Pirineos, 153-154: 21 a 59, JACA; 1999

\title{
ESTRUCTURA DE UN ECOTONO BOSQUE SUBALPINO-PASTOS ALPINOS (LAS CUTAS, ORDESA, PIRINEOS CENTRALES)
}

\author{
J. J. Camarero \& E. Gutiérrez \\ Departamento de Ecología, Facultad de Biología, Universidad de Barcelona. \\ Avda. Diagonal, 645. 08028 Barcelona. España. \\ Tel. 4021516. Fax 934111438 \\ C. e.: chechu@porthos.bio.ub.es, emilia@porthos.bio.ub.es
}

RESUMEN.- Describimos la estructura de un ecotono bosque subalpino-pastos alpinos en los Pirineos Centrales, que incluye los limites altitudinales del bosque y del árbol y está dominado por Pinus uncinata Ram. Para cada individuo de P. uncinata situado dentro de una parcela rectangular paralela a la pendiente anotamos su localización y medimos diversas variables estructurales o de forma (número y tipo de pies por individuo). Estos individuos fueron clasificados según su tamaño (adultos, jóvenes, vástagos y plántulas) y forma («krummholz» —individuos arbustivos policórmicos-, krummholz con pies verticales). La estructura del ecotono se describió mediante los cambios de tamaño y forma de los individuos de P. uncinata a lo largo del ecotono y en relación con el clima de la zona, que se caracteriza por espesores de nieve máximos en primavera (abril) y fuertes vientos del N-NW-W. La mayoría de los individuos vivos eran krummholz, "situándose por encima del límite del bosque y mostrando proximidad espacial con las plántulas. Los individuos grandes, verticales y unicórmicos predominaban en el bosque. Los cambios estructurales o de forma de los individuos eran bruscos a lo largo del ecotono. Los daños de las copas debidos al viento eran evidentes en individuos arbustivos y predominaban en las direcciones de viento más fuertes durante todo el año. La interacción nieve-viento permite explicar en parte la localización de este limite del bosque que puede considerarse un fenómeno local. La situación espacial de las distintas clases de individuos, la interacción espacial entre plántulas y krummholz más los cambios de forma de crecimiento (de arbustiva a arbórea o viceversa) pueden modificar la respuesta de estas poblaciones de P. uncinata a cambios climáticos.

SUMMARY.- We describe the structure of a subalpine forest-alpine pasture ecotone in the Central Pyrenees, that includes altitudinal timberline and treeline, and it is dominated by Pinus uncinata Ram. We measured for each P. uncinata 


\section{PIRINEOS 153-154}

individual located inside a rectangular plot parallel to the slope: the location and several structural and growth form variables (number and type of stems per individual). Then, P. uncinata individuals were classified according to their size (adults, poles, saplings and seedlings) and growth form (krummholz - shrubby and multistemmed individuals-, krummholz with vertical stems). The ecotone structure was described through the study of growth form and size changes of P. uncinata individuals across the ecotone and related to the area climate, that is characterized by maximum snow thickness in spring (april) and strong N-NW-W winds. Most of living individuals were krummholz, located above the timberline in the area where seedlings were abundant. Bigger, vertical and unistemmed individuals predominated in the forest. The structure or growth form changes were sharp across the ecotone. Crown injuries due to wind effects were evident in shrubby individuals and predominated in those directions that showed stronger winds during the year. The snow-wind interaction can explain partially the location of the studied timberline that could be considered a local phenomenon. The spatial location of the different classes of individuals, the spatial interaction between seedlings and krummholz individuals, and the changes of growth form (from arborescent to shrubby or vice versa) can modify the response of these $P$. uncinata populations to climate changes.

RÉSUMÉ.- Nous avons analysé la structure d'un écotone marqué par le passage de la forêt subalpine à la prairie alpine dans les Pyrénées Centrales. Cet écotone incluant les limites altitudinales de la forêt et des arbres est dominé par le Pinus uncinata Ram. A l'intérieur d'une parcelle rectangulaire parallèle à la pente, nous avons mesuré la position de chaque tige de $\mathrm{P}$. uncinata ainsi que plusieurs paramètres décrivant la structure et la forme des arbres (abondance et type de tige par individus). Nous avons classifié les individus de ce pin selon leur taille (adulte, "pole», gaulis et semis) et leur forme de croissance (krummholz -à tiges multiples et à port arbustif-, krummholz à tiges verticales). La structure de l'écotone a été decrite par l'analyse des formes de croissance et de la taille des individus de P. uncinata et celle-ci fut a été reliée aux particularités du climat régional lequel se caractérise par une accumulation maximale de la neige au printemps (avril) et la dominance des vents du N-NW-W. La majorité des individus vivants étaient constitués de krummholz et se retrouvaient au delà de la limite altitudinale de la forêt, a l'endroit même où les semis abondaient. Les individus de fort diamètre, à tige verticale et unique dominaient surtout dans l'étage forestier. Les changements dans la structure ou les formes de croissance étaient très marqués le long de l'écotone. Les dommages à la cime causés par les vents étaient frequents chez les individus à port arbustif et prédominaient selon l'orientation des vents dominants. L'interaction entre la direction des vents et l'accumulation de la neige au sol peut expliquer, en partie, la localisation actuelle de la limite des arbres dans l'écotone étudié. La position de l'écotone semble être le reflet des conditions locales. La localisation spatiale des différentes classes d'individus, l'interaction spatiale entre les semis et les krummholz, de même que les changements dans le port des arbres (arborescent à arbustif et vice versa) peuvent modifier la réponse des populations de P. uncinata aux changements climatiques.

Key-words: ecotone, treeline, timberline, Pinus uncinata, growth-form, krummholz, size structure, spatial pattern. 
ESTRUCTURA DE UN ECOTONO BOSQUE SUBALPINO-PASTOS ALPINOS..

\section{Introducción}

El cambio global, que incluye el cambio climático así como modificaciones en usos del suelo y en los ciclos biogeoquímicos (VITOUSEK, 1994), puede afectar de forma significativa a las poblaciones de árboles situadas en sus límites de distribución tales como el ecotono formado por los límites altitudinales del bosque (timberline o forest limit) y del árbol (treeline) (BRUBAKER, 1986; PAYETTE \& LAVOIE, 1994). Por otro lado, los ecotonos son considerados zonas teóricamente sensibles a cambios ambientales aunque falta consenso sobre la escala espacial a estudiar o cómo detectar y medir esa teórica sensibilidad (HANSEN \& DI CASTRI, 1992).

Se ha constatado la existencia de un equilibrio dinámico entre el clima y el límite del árbol a una escala temporal superior a los 100 años y en áreas como Escandinavia, donde la historia de perturbaciones humanas es larga (KULLMAN, 1979, 1991). No obstante, otros autores han resaltado la influencia de factores antrópicos como el pastoreo (ovejas y renos), factores edáficos y su interacción con el clima sobre el dinamismo del límite del bosque latitudinal (OKSANEN et al., 1995). Existen muchos trabajos sobre regiones de Norteamérica, donde la presión antrópica fue menor siglos antes que en Eurasia, documentando la invasión de prados subalpinos por coníferas, el crecimiento vertical de individuos arbustivos (krummholz) y el establecimiento de plántulas sobre el límite del bosque durante este siglo - aunque no la ascensión altitudinal de este límite-(BRINK, 1959; FRANKLIN et al., 1971; DUNWIDDIE, 1977; KEARNEY, 1982; JAKUBOS \& ROMME, 1993). Estos estudios se han realizado en zonas fuera o dentro de Parques Nacionales y sus resultados han generado controversias sobre la gestión de dicho ecotono (VALE, 1977, 1987; IVES \& HANSEN-BRISTOW, 1983). En el Pirineo Central, ciertos datos (fotografías aéreas, datos documentales) sugieren la posible ascensión altitudinal del límite del bosque subalpino durante los últimos 50 años (SOUTADÉ et al., 1982), lo cual podría deberse en parte a condiciones climáticas favorables y al descenso reciente de la presión ganadera (GARCÍA RUIZ \& LASANTA MARTÍNEZ, 1990; BAS, 1993).

Nuestro objetivo será la descripción estructural del ecotono formado por el límite del bosque subalpino de $P$. uncinata y los pastos alpinos en la Sierra de las Cutas, Ordesa. Esta descripción es un paso previo para inferir los procesos y la dinámica que generaron dicha estructura (mediante estudios dendroecológicos) y decidir si, en el ecotono estudiado, se está produciendo recolonización o invasión de nuevo terreno por parte de P. uncinata. 


\section{Metodología y área de muestreo}

\subsection{Situación y especie}

El límite del bosque del pinar de la Carquera está en la Sierra de las Cutas, zona de preparque del Parque Nacional de Ordesa y Monte Perdido (Lat. $42^{\circ}$ $37^{\prime} \mathrm{N}$, Long. $00^{\circ} 01^{\prime} \mathrm{W}$ ). La altitud y pendientes medias son $2100 \mathrm{~m}$ y $17^{\circ}$ (pendiente máxima de $33^{\circ}$ en el bosque), respectivamente. La orientación es de $186^{\circ}$ S-SW. La selección de una zona de preparque se basó en el supuesto teórico de una reducción durante el último siglo de perturbaciones humanas (sobrepastoreo, talas e incendios), ya que los Pirineos muestran límites del bosque muy alterados por actividades humanas locales. Las altitudes máximas de los límites del bosque, del árbol —considerando árbol todo individuo de $P$. uncinata con al menos $2 \mathrm{~m}$ de altura; KULLMAN, 1979- y de los individuos krummholz fueron $\sim 2100 \mathrm{~m}, 2110 \mathrm{~m}$ y $2150 \mathrm{~m}$, respectivamente. En esta misma zona, GIL PELEGRÍN (1993) realizó estudios ecofisiológicos sobre los individuos krummholz y consideró este límite como local y poco afectado por perturbaciones humanas recientes (GIL PELEGRÍN \& VILLAR, 1988).

En las Cutas se producen contactos entre margas con algunas turbiditas y margocalizas del Eoceno, y aparecen calizas masivas y areniscas de cemento silíceo del Paleoceno (Terciario) en la zona de la Carquera. Los suelos son mayoritariamente básicos y derivados de flysch areniscoso sometido al ciclo de hielo y deshielo aunque las areniscas originan sustratos ácidos en ciertas zonas (RÍOS et al., 1982).

Durante el Tardiglaciar, el límite del bosque pirenaico estaba localizado a una altitud inferior a los $1700 \mathrm{~m}$ en los Pirineos Centrales aragoneses (MONTSERRAT, 1992). No todos los ecotonos bosque subalpino-pastos alpinos pirenaicos siguen la típica secuencia de formas de crecimiento desde árboles verticales y de un sólo pie (unicórmicos) próximos al límite del bosque o dentro de él hasta individuos arbustivos policórmicos determinados fenotípicamente (krummholz, en sentido amplio) y situados a mayor altitud que el límite del árbol (GIL PELEGRÍN \& VILLAR, 1988).

En los Pirineos, P. uncinata predomina en la mayoría de los límites forestales y arbóreos altitudinales (CANTEGREL, 1983). P. uncinata coloniza todo tipo de suelos, es una especie pionera y heliófila (CEBALLOS \& RUIZ de la TORRE, 1979; BOSCH et al., 1992). Su reproducción es predominantemente sexual ya que el viento dispersa la mayoría de sus ligeras semillas, de entre las más pequeñas de todas las especies de pinos europeos (MIROV, 1967). Sus plántulas son muy resistentes a las heladas incluso con escasa protección nival (FREY, 1983). Para ilustrar alguno de los resultados, se compararán tres límites altitudinales del bosque pirenaico dominados por P. uncinata: el aquí estu- 
diado situado en Las Cutas-Ordesa (Sobrarbe, Huesca), Tessó del Son-Son del Pí (cerca del Parque Nacional de Aigüestortes i Estany de St. Maurici, Pallars Sobirà, Lleida) y Estanys de la Pera-Lles de Cerdanya (Cerdanya, Girona).

\subsection{Clima}

La región de estudio pertenece a zonas de alta montaña (> 1500 m s.n.m.), más frías y lluviosas que las áreas circundantes y con presencia habitual de heladas (ALLUÉ ANDRADE, 1990). Fitoclimáticamente, estas zonas resumen, a lo largo del gradiente altitudinal, las transiciones latitudinales que suceden a una escala geográfica mayor. En la Sierra de las Cutas, el clima puede considerarse continental con influencia mediterránea de alta montaña. La mejor estación para describir nuestra zona de estudio es el Refugio de Góriz (2215 m, 42 $39^{\circ} \mathrm{N}, 0^{\circ} 01^{\prime} \mathrm{E}$ ) que dispone de pocos años de registro (usamos datos de 1981-89 y 1993-95). Disponemos también de datos diarios desde diciembre de 1992 hasta abril de 1995 inclusive. Al comparar dos diagramas ombroclimáticos de estaciones pirenaicas de alta montaña (Pic du Midi, datos de BÜCHER \& DESSENS, 1991; Estany Gento, datos de MONTERO \& GONZÁLEZ, 1974), observamos que sólo la más elevada y occidental posee una temperatura media de julio inferior a $10{ }^{\circ} \mathrm{C}\left(6,1^{\circ} \mathrm{C}\right.$, Figura 1$)$, siendo la altitud de esta estación superior a los límites actuales forestal y del árbol más elevados en los Pirineos ( $2500 \mathrm{~m}$ para el bosque y $2740 \mathrm{~m}$ para individuos aislados de P. uncinata; VIGO, 1976; BOLÒS et al., 1993; CARRERAS et al., 1996). De las tres estaciones comparadas, la que posee una temperatura media de julio más próxima a $10^{\circ} \mathrm{C}$ es Estany Gento $\left(11,7^{\circ} \mathrm{C}\right)$, situada a una altitud de $2174 \mathrm{~m}$, frente a Góriz con valores más elevados de altitud $(2215 \mathrm{~m}$ ) y temperatura media de julio $\left(13,3^{\circ} \mathrm{C}\right)$, lo cual reforzaría la hipótesis sobre el carácter local del límite del bosque de las Cutas. Aunque Pic du Midi posee un largo registro de precipitaciones totales mensuales (1882-1984), sus datos muestran heterogeneidad y discontinuidades desde 1937 y no son fiables desde 1923 (DESSENS \& BÜCHER, 1997). Si seleccionamos el periodo válido de precipitaciones para Pic du Midi (1882-1922, 41 años), aparece un máximo invernalprimaveral de precipitaciones y un claro mínimo estival-otoñal, mientras que Góriz y Estany Gento presentan máximos primaveral y otoñal (Figura 1).

La temperatura media anual es $4,7^{\circ} \mathrm{C}$ y las temperaturas medias mensuales máxima y mínima son $13{ }^{\circ} \mathrm{C}$ (julio) y $-1,92{ }^{\circ} \mathrm{C}$ (febrero), existiendo tres meses (enero, febrero y marzo) con temperaturas medias mensuales inferiores a $0^{\circ} \mathrm{C}$ y otros tres (julio, agosto y septiembre) con temperaturas medias iguales o superiores a $10^{\circ} \mathrm{C}$ (Figura 1). La media de las máximas más elevada corresponde a julio $\left(16,4^{\circ} \mathrm{C}\right)$ y la menor a febrero $\left(1,8{ }^{\circ} \mathrm{C}\right)$. Respecto a las 


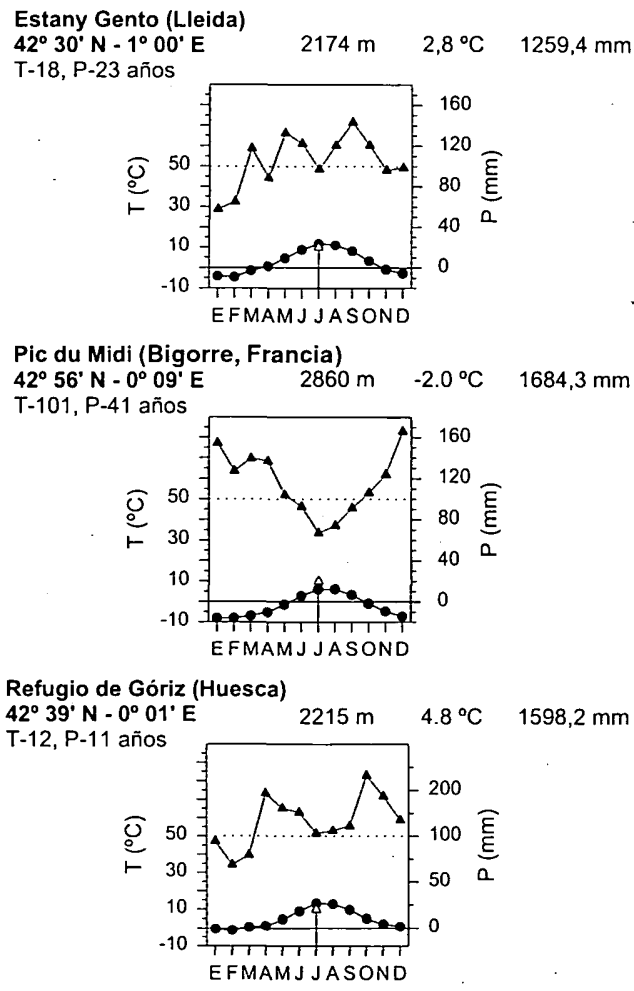

Figura 1. Diagramas ombrotérmicos (círculos, temperatura media mensual; triángulos, precipitación total mensual) para la estación del Refugio de Góriz (datos de 1982-94, figura inferior) en comparación con otras estaciones de alta montaña situadas en los Pirineos centrales (Pic du Midi, Francia, datos de temperatura para los años 1882-1984, excepto 1943-44, y de precipitación para el periodo 1882-1922; Estany Gento, datos de ALLUÉ ANDRADE, 1990). La estación de Góriz muestra un carácter más oromediterráneo con una mayor temperatura media anual a pesar de su mayor altitud respecto a Estany Gento. En todos los diagramas se señala la temperatura media para julio igual a $10^{\circ} \mathrm{C}$ (triángulo blanco), isoterma próxima al límite del bosque (GRACE, 1989). Obsérvese el cambio de escala para las precipitaciones, en el caso de valores totales mensuales superiores a los 150 $\mathrm{mm}$ para las estaciones de Pic du Midi y Góriz.

Climate diagrams (circles, mean monthly temperature; triangles, total monthly precipitation) for the Refugio de Góriz station (1982-94 data, lower figure) and other elevated stations located in the Central Pyrenees (Pic du Midi, France, temperature data for the period 1882-1984, excepting years 1943-44, and precipitation data for the period 1882-1922; Estany Gento, data from ALLUÉ ANDRADE, 1990). Góriz station shows a more oromediterranean character with a greater annual mean temperature in spite of being located at a higher elevation than Estany Gento. The value of $10^{\circ}$ $\mathrm{C}$ for the July mean temperature is shown in all diagrams (white triangle), because the isotherm for this value is considered a good approximation of the theoretical timberline (GRACE, 1989). There is a change of scale for the total monthly precipitation data in the case of values greater than $150 \mathrm{~mm}$ for the Pic du Midi and Góriz stations. 
medias de las mínimas, la mayor y la menor pertenecen de nuevo a julio $(9,6$ $\left.{ }^{\circ} \mathrm{C}\right)$ y febrero $\left(-5,6^{\circ} \mathrm{C}\right)$, respectivamente. Durante el período $1981-89$, las máxima y mínima temperaturas absolutas registradas en el Refugio de Góriz fueron $25,5^{\circ} \mathrm{C}$ (agosto 1987) y $-21,0^{\circ} \mathrm{C}$ (enero 1985). Los meses con mayores promedios de días de helada son, en orden decreciente: marzo (27 días), febrero y enero (25 días en ambos casos). La media de días de helada durante el año es alta, alcanzando 161 días (BALCELLS \& GIL PELEGRÍN, 1992). Las temperaturas mínimas absolutas del aire fueron, en promedio $( \pm \mathrm{SD})$, más bajas $\left(-9,6 \pm 4,0^{\circ} \mathrm{C}\right)$ que aquellas tomadas en la superficie de la nieve $\left(-7,7 \pm 3,6^{\circ} \mathrm{C}\right)$ para los meses con innivación desde enero de 1992 hasta abril de 1995, aunque dichas diferencias no son significativas (test de Mann-Whitney, $\mathrm{p}=0,19$, $n=17$ ). La isoterma para la media de las temperaturas mínimas del mes más cálido (suele ser julio en estas latitudes) se ha situado en el Pirineo Central a unos 2450 m (CREUS, 1978; BARRIO et al., 1990). Esta isoterma se ha considerado una aproximación bioclimática potencial al límite superior del árbol o treeline (TRANQUILLINI, 1979; TUHKANEN, 1980; GRACE, 1989).

La precipitación anual estimada es próxima a los $1600 \mathrm{~mm}$, con una gran variabilidad interanual (p. ej. se recogieron $2900 \mathrm{~mm}$ en 1987-88 frente a los 1300 mm de 1985-86 o 1988-89). La precipitación se distribuye mayoritariamente en otoño $(32 \%)$ y primavera $(30 \%)$, con mínimos estivales $(22 \%)$ y, sobre todo, invernales (16\%). La precipitación mensual total tiene máximos en octubre $(\approx 200 \mathrm{~mm})$ y febrero-marzo, y mínimos $(<100 \mathrm{~mm})$ estivales durante agosto-septiembre.

El espesor medio ( \pm SD) de nieve fue $36,6 \pm 21,7 \mathrm{~cm}$ durante la década de los 80. Las precipitaciones nivales son mayores en primavera (abril, p. ej. 1993) que en invierno (Figuras 2 y 3), lo cual podría mejorar las reservas hídricas para la iniciación del crecimiento vegetal a finales de primavera y acortar o interrumpir el período vegetativo retrasando su inicio (BALCELLS \& GIL PELEGRÍN, 1992). Tanto la precipitación total mensual en forma de nieve como el número de días por mes con el suelo cubierto por nieve son significativamente mayores $(p<0,05, n=29)$ para las mediciones matinales ( $8 \mathrm{~h})$ que para aquellas tomadas pasado el mediodía (13 h, Figura 3$)$. El espesor máximo invernal de nieve puede alcanzar los 150-200 cm, sin embargo la precipitación invernal en forma de nieve puede ser nula. La innivación más constante sucede en primavera (abril) y en pleno invierno, siendo este último período el más frío y uno de los más secos en cuanto a precipitaciones. El fin de la innivación oscila entre finales de mayo y principios de junio. El promedio de días innivados por año es un 48\% (BALCELLS \& GIL PELEGRÍN, 1992).

Para los datos diarios de Góriz usados (diciembre 1992-abril 1995), encontramos una correlación significativa (coeficiente de correlación de Spearman, $r_{s}=0,29, p<0,001, n=501$ ) y positiva entre la diferencia de espesores de nieve 


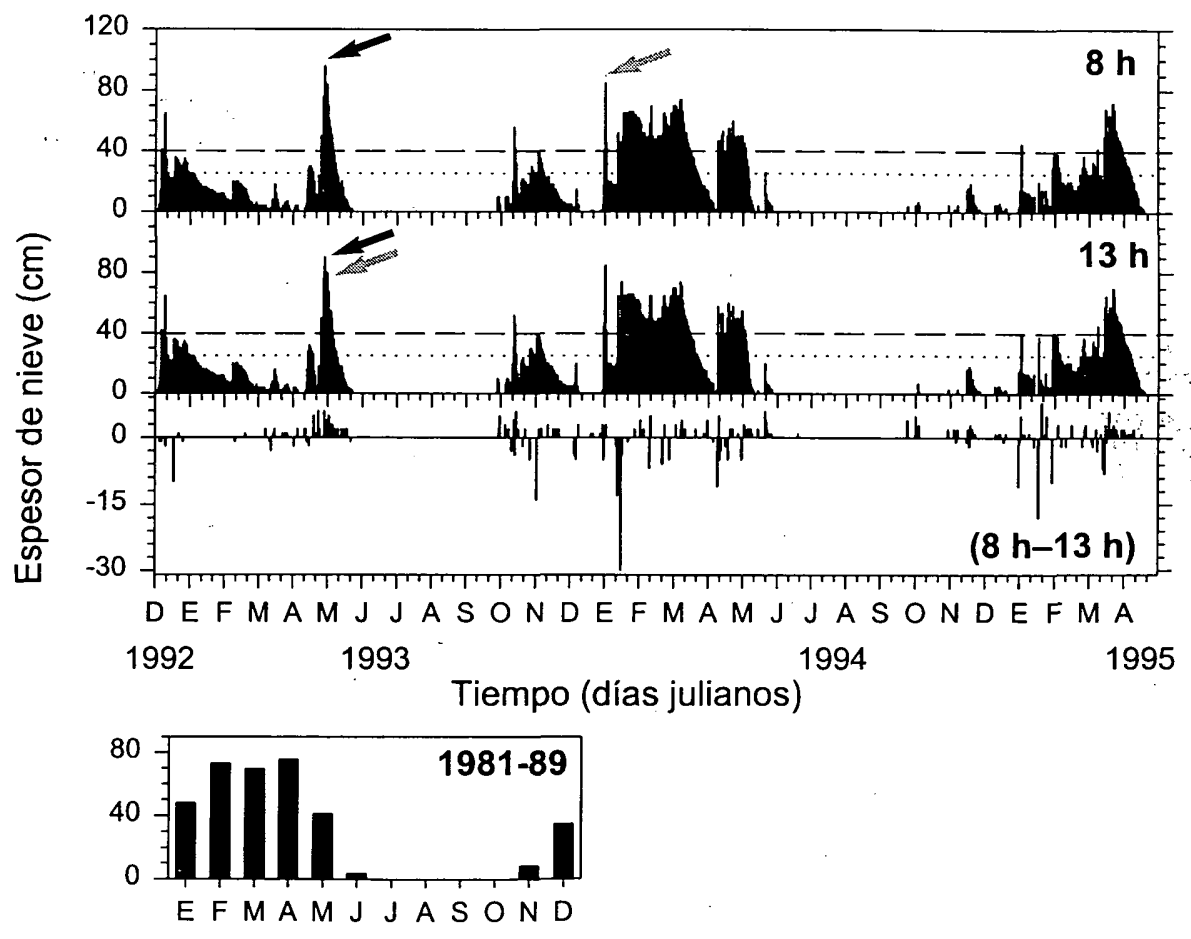

Figura 2. Espesor total diario de nieve $(\mathrm{cm})$ en el observatorio meteorológico del Refugio de Góriz. Se muestran las mediciones tomadas a las 8 y 13 h desde diciembre de 1992 hasta abril de 1995 así como el valor medio (línea a puntos) y el doble de la desviación estándar (línea discontinua) para los días con presencia de nieve en el suelo. La tercera figura inferior ( $8 \mathrm{~h}-13 \mathrm{~h})$ muestra las diferencias entre el espesor medido a las $8 \mathrm{~h}$ y el medido a las $13 \mathrm{~h}$ para aquellos días con mediciones a ambas horas. Las flechas indican los espesores máximos (flechas negras) y los segundos espesores máximos (flechas grises) para el período señalado. La figura inferior pequeña muestra el espesor promedio de nieve en meses para el período 1981-89, según datos de BALCELLS \& GIL PELEGRIN (1992).

Total daily snow thickness $(\mathrm{cm})$ measured in the Refugio de Góriz meteorological station. The measurements made at 8 and $13 \mathrm{~h}$ from December 1992 to April 1995, their mean value (dotted line) and the standard deviation multiplied by 2 are shown (dashed line). These last two values were calculated considering only those days with presence of snow in the soil. The third lower figure ( $8 h-13 h)$ shows the difference between the snow thickness measured at 8 and $13 \mathrm{~h}$ for those days with measurements taken at both times. The arrows indicate the maximum thickness (black arrows) and the second greatest thickness (gray arrows) for all the period. The small lower figure shows the mean monthly snow thickness for the 1981-89 period, according to data from BALCELLS \& GIL PELEGRÍN (1992).

$(\mathrm{cm})$ medidos a las $8 \mathrm{~h}$ y a las $13 \mathrm{~h}$ (nieve presente en la mañana menos nieve presente pasado el mediodía o disminución del espesor de nieve al avanzar el día, Figura 2) y la temperatura media del aire a las $13 \mathrm{~h}$. Esta correlación fue 

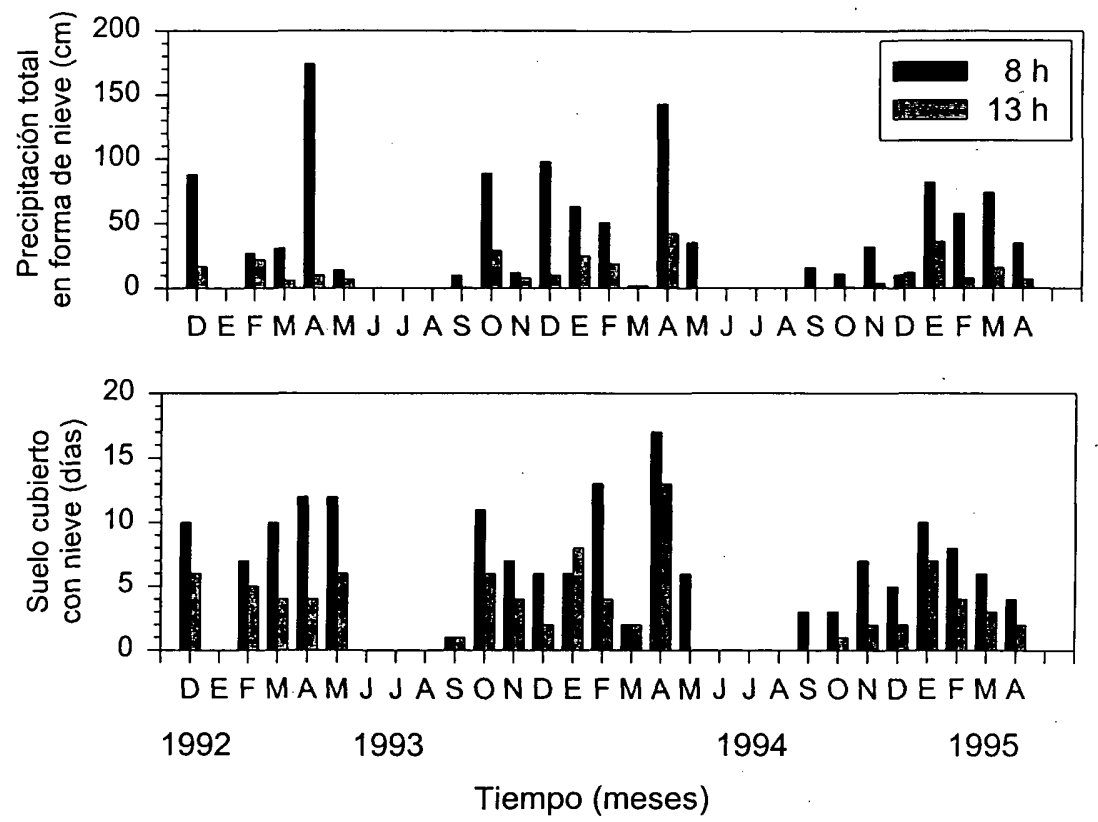

Figura 3. Precipitación total mensual en forma de nieve $(\mathrm{cm})$ y número de días por mes en los que el suelo aparece cubierto por nieve (datos del obervatorio del Refugio de Góriz promediados para las mediciones diarias tomadas a las 8 y $13 \mathrm{~h}$ desde diciembre de 1992 hasta abril de 1995). Total monthly precipitation recorded as snow $(\mathrm{cm})$ and number of days per month with presence of snow in the soil (the data are from the Refugio de Goriz station and have been averaged for the daily measurements made at 8 and $13 \mathrm{~h}$ from December 1992 to April 1995).

significativa también con la diferencia de temperaturas medias registradas a las $13 \mathrm{~h}$ y $8 \mathrm{~h}$ que equivale al aumento de temperatura media al avanzar el día $\left(r_{s}=0,21, p<0,001, n=502\right)$. El espesor de nieve presente a las $13 \mathrm{~h}$ estaba significativa y negativamente correlacionado con las temperaturas mínima $\left(r_{s}=\right.$ $-0,25, p<0,001, n=502)$ y máxima $\left(r_{s}=-0,16, p<0,001, n=500\right)$ medidas a las $8 \mathrm{~h}$ así como con la media registrada a las $13 \mathrm{~h}\left(r_{s}=-0,15, p<0,01, n=501\right)$. Similares resultados se obtuvieron para el espesor de nieve medido a las $8 \mathrm{~h}$, estando más determinado por la temperatura mínima de las $8 \mathrm{~h}\left(r_{s}=-0,24, p\right.$ $<0,001, n=502)$ que por la máxima a la misma hora $\left(r_{s}=-0,14, p<0,01, n=\right.$ $500)$. En resumen, días de mañanas con temperaturas mínimas altas y mediodías cálidos reducen el espesor de nieve presente después del mediodía. Sin embargo, y a modo de ejemplo, el 9 de enero de 1994 se produjo un aumento de $30 \mathrm{~cm}$ del espesor de nieve medido a las $13 \mathrm{~h}$ respecto del presente a las $8 \mathrm{~h}$ 
causado por una nevada importante (Figura 2), coincidiendo con una elevación de la temperatura media desde los $-2,8{ }^{\circ} \mathrm{C}$ a las $8 \mathrm{~h}$ hasta los $2{ }^{\circ} \mathrm{C}$ a las 13 h y con temperaturas no muy frías para la época del año.

Los dos vientos predominantes en Aragón son el cierzo y el bochorno que provienen del N-NW y del S-SW, respectivamente (Figuras 4 y 5). El cierzo es un viento muy fuerte, glacial en invierno pero desecante en la vertiente pirenaica sur durante el verano. En años en que coinciden la dominancia del cierzo y el anticiclón del Ebro, las borrascas atlánticas son desviadas hacia vertientes septentrionales donde descargan generando viento seco descendente a sotavento en las laderas meridionales (solanas). Esto provoca el máximo primaveral de innivación, época en que las borrascas superan el efecto anticiclónico (BALCELLS \& GIL PELEGRÍN, 1992). Otros años de características climáticas continentales, la nieve es abundante (cada tres años aproximadamente) y permanece desde otoño hasta primavera con un máxi-

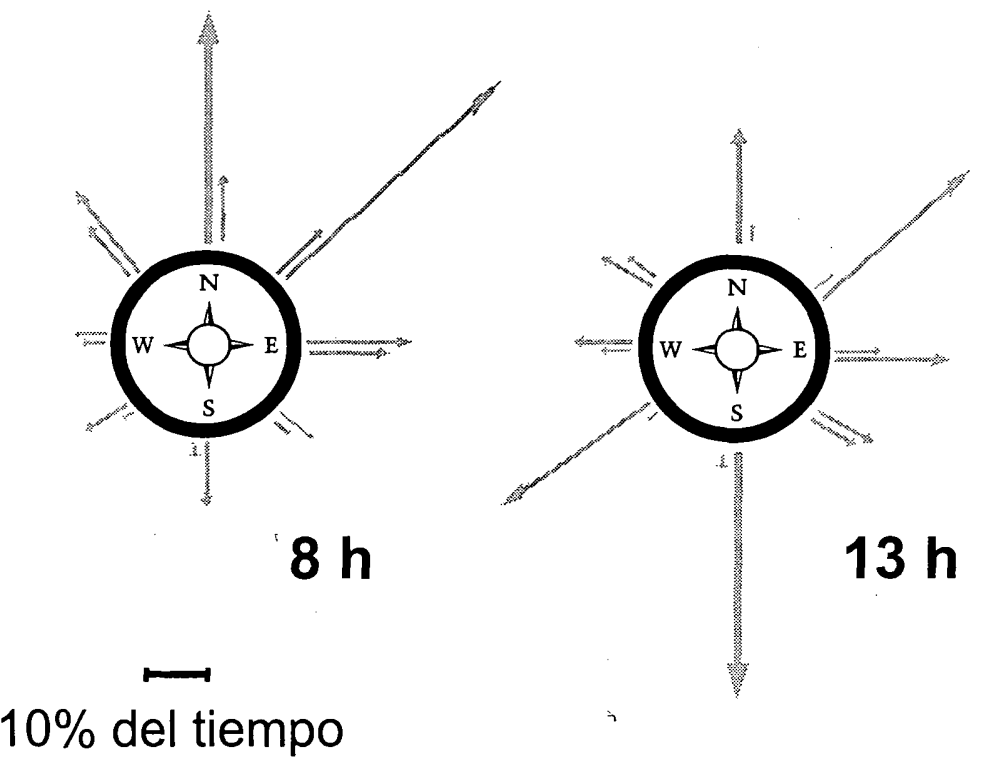

Figura 4. Dirección de procedencia del viento (porcentaje de días durante el período diciembre 1992-abril 1995) durante todo el período (flechas negras) o sólo en invierno (noviembre-abril, flechas grises) registrada a las 8 y 13 h. Datos del Refugio de Góriz. La barra inferior muestra la escala ( $10 \%$ del tiempo).

Wind direction (percentage of days during the period December 1992-April 1995) for all the period (black arrows) or only for winter (November-April, gray arrows) recorded at 8 and $13 \mathrm{~h}$. Data from Refugio de Goriz station. The lower bar shows the scale (10\% of time). 


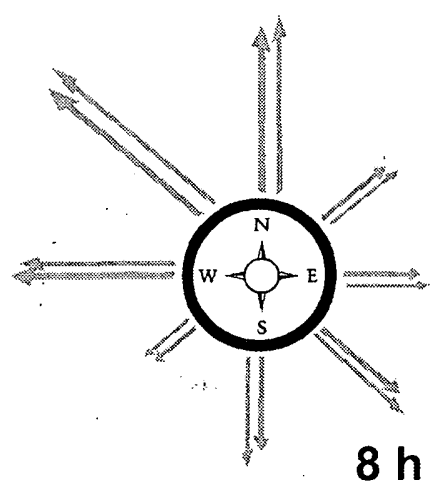

$2 \mathrm{~m} \mathrm{~s}^{-1}$

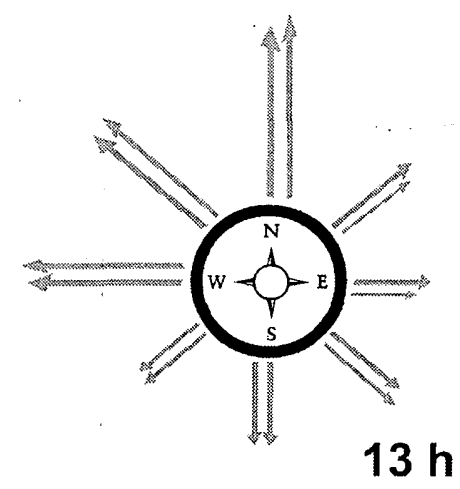

$13 \mathrm{~h}$

Figura 5. Velocidad media del viento $\left(\mathrm{m} \cdot \mathrm{s}^{-1}\right)$ procedente de distintas direcciones durante el período diciembre 1992-abril 1995 (flechas negras) y sólo en los inviernos (noviembre-abril) de este período (flechas grises) medida a las 8 y $13 \mathrm{~h}$. Datos del Refugio de Góriz. La barra inferior muestra la escala $\left(2 \mathrm{~m} \cdot \mathrm{s}^{-1}\right)$.

Mean wind velocity $\left(\mathrm{m} \cdot \mathrm{s}^{-1}\right)$ is shown for different directions for the period December 1992-April 1995 (black arrows) and only for winter (November-April, gray arrows). The measurements were made at 8 and $13 \mathrm{~h}$. Data from Refugio de Góriz station. The lower bar shows the scale $\left(2 \mathrm{~m} \cdot \mathrm{s}^{-1}\right)$.

mo invernal. Durante el período comprendido entre diciembre de 1992 y abril de 1995, las dos velocidades máximas del viento medidas a las $8 \mathrm{~h}$ en el Refugio de Góriz fueron $92,6 \mathrm{~km} \cdot \mathrm{h}^{-1}$ (2 de marzo de 1995, dirección de procedencia NW) y $74,1 \mathrm{~km} \cdot \mathrm{h}^{-1}$ (9 de diciembre de 1992, dirección N). Al final del invierno, el fuerte viento puede dejar sin nieve muchas solanas de altitud elevada (1950-2200 m). Los vientos más frecuentes soplan del $\mathrm{N}$ por la mañana y del $S$ a partir del mediodía, aunque en invierno predominan los de componente NE, NW y, pasado el mediodía, del SW (Figura 4). Tanto durante el año como en invierno, los vientos más fuertes suelen soplar del N-NW y, a medida que avanza el día, del W (Figura 5). Esto puede perjudicar a algunas plantas leñosas subalpinas como el rododendro al «limpiar» amplias zonas de nieve y privar a los individuos de la protección conferida por la nieve frente a la desecación y el daño físico de abrasión producido por los fuertes vientos que arrastran cristales de hielo (HADLEY \& SMITH, 1983). En el Refugio de Góriz y durante el período desde diciembre de 1992 hasta abril de 1995, la velocidad diaria ( \pm 1 SD) del viento medida a las $8 \mathrm{~h}(17,6 \pm$ $\left.13,5 \mathrm{~km} \cdot \mathrm{h}^{-1}\right)$ fue significativamente $(p<0,05, n=512)$ mayor que la medida a las $13 \mathrm{~h}\left(15,1 \pm 10,7 \mathrm{~km} \cdot \mathrm{h}^{-1}\right)$. 


\subsection{Flora}

Nos encontramos en el límite de un pinar oromediterráneo de $P$. uncinata típico de solanas elevadas (1600-2200 m), con presencia de Juniperus communis subsp. nana y Arctostaphylos uva-ursi en el sotobosque y dominado por pastos compuestos por la calcícola Festuca gautieri (VILLAR \& BENITO ALONSO, 1996; VILLAR \& MONTSERRAT RECODER, 1990). Estas comunidades se caracterizan por situarse en zonas de elevada oscilación térmica, máximos primaverales de innivación, inviernos con valores pequeños de espesor de nieve o incluso sin cobertura de nieve, elevada insolación, sustrato básico y están compuestas por especies de las regiones eurosiberiana ( $83 \%$ de las alianzas botánica del parque) y mediterránea ( $16 \%$ de las alianzas) (VILLAR \& BENITO ALONSO, 1994). Por degradación debida a sobrepastoreo e incendios derivan a matorrales de Juniperus communis o Echinospartum horridum. En Ordesa, estas comunidades aparecen en solanas calizas como las Cutas o la Larri en Pineta (VILLAR \& BENITO ALONSO, 1994). En los claros del pinar de las Cutas (1870 m), ALDEZÁBAL et al. (1992) encontraron en el sotobosque individuos de Rhododendron ferrugineum, Vaccinium myrtillus, Calluna vulgaris, Festuca gautieri, Anthoxanthum odoratum y Nardus stricta, entre otras especies. Algunas de estas especies son acidófilas ( $N$. stricta p. ej.) debido a la presencia de areniscas.

\subsection{Uso histórico: pastoreo y perturbaciones}

La población rural pirenaica ha disminuido de manera general a lo largo del siglo xx debido a la emigración, lo que ha conllevado el abandono de tierras y ha permitido, en parte, el descenso de la cabaña ganadera ovina desde aproximadamente 1950 (GARCÍA RUIZ \& LASANTA MARTÍNEZ, 1990; CHOCARRO et al., 1990; BAS, 1993).

Las antiguas zonas de pasto de los pueblos de Fanlo, Buisán y Ceresuela se situaban desde la Punta Acuta hasta el Barranco de Calderuela siendo el límite superior la Sierra Acutas (M. ETXEBARRÍA, com. pers.). La mayor concentración ganadera se daba en el Lomar, situado en la parte N-NW del valle, en cuya zona superior se sitúa el pinar de La Carquera cuyo límite altitudinal estudiamos. Hasta 1950, el número aproximado de cabezas en el. valle del Jalle era 9000 de ovino y 60 de vacuno, más 60 de caballar. El número actual de cabezas ronda las 3000-3500 de ovino y unas 130 de vacuno (ALDEZÁBAL et al., 1992), lo que supondría una disminución del $64 \%$ de ganado ovino pero un $217 \%$ de aumento del ganado vacuno, siguiendo tendencias generales para el Pirineo aragonés (GARCÍA RUIZ \& LASANTA MARTÍNEZ, 1990). Según testimonios de vecinos de Fanlo, el pinar de La 
Carquera no habría cambiado sus dimensiones a lo largo de este siglo (M. ETXEBARRÍA, com. pers.).

La solana de Fanlo está actualmente aprovechada por el pequeño rebaño de cabras de Torla en cotas altas (2100-2200 m) y por ganado ovino principalmente (2600 cabezas de ovino y 130 vacas) de Fanlo y Buisán que asciende en primavera y otoño (ALDEZÁBAL et al., 1992). Nuestra zona de estudio (altitud $2100 \mathrm{~m}$, fuerte insolación) sería principalmente pastoreada por ovejas, cabras y sarrios, teniendo en cuenta que estos autores no encontraron cargas ganaderas elevadas en la zona del parque y periferia (0,7 -3,4 Unidades de Ganado Mayor mes $\cdot$ ha $^{-1}$, donde 1 U.G.M = 8-10 ovejas y 0,8 U.G.M. = 1 vaca). Estos autores reconocen el descenso del número de herbívoros domésticos y recomiendan el mantenimiento de cargas ganaderas mínimas para la «conservación del ecosistema pastoral», aunque citan el efecto negativo del pastoreo excesivo sobre las especies de plantas endémicas. Sin embargo, reconocen la falta de estudios sobre las tendencias futuras en los ecosistemas pastorales y forestales tras la disminución del pastoreo.

Sólo 14 individuos arbustivos de P. uncinata (krummholz) situados próximos al límite del árbol mostraron signos de pastoreo. También observamos marcas de teas de pastores en dos individuos arbóreos en el límite inferior de la parcela. Al comparar fotografías aéreas recientes (1946, E. 1:43.000; 1957, E. 1:32.000; 1986-88 E. 1:5.000) no se observa ningún desplazamiento altitudinal del límite del bosque aunque sí cierto aumento de su densidad. En los años 30 un incendio afectó al pinar de la Carquera en cotas inferiores al límite del bosque $(2080 \mathrm{~m})$, lo que atestiguan algunos restos de tocones quemados.

\subsection{Muestreo}

El muestreo se realizó en una parcela rectangular $(140 \mathrm{~m}$ x $30 \mathrm{~m})$ situada cruzando el ecotono pastos alpinos-bosque en un lugar de topografía homogénea y representativo del ecotono, con su lado más largo paralelo a la máxima pendiente y localizando sus límites superior e inferior próximos e incluyendo los límite del árbol y del bosque (límite inferior ya dentro del bosque), respectivamente. Cartografiamos la posición de todos los individuos de $P$. uncinata siguiendo dos ejes cartesianos paralelos a los ejes menor $(x)$ y mayor de la parcela $(y)$. El punto $(x, y)=(0,0)$ se situó en la esquina superior derecha mirando hacia arriba. La parcela fue subdividida con otras cintas métricas para medir las coordenadas de la posición de los árboles con una precisión de $\pm 0,1 \mathrm{~m}$. El muestreo se realizó durante julio-septiembre de 1995.

Para cada individuo localizado de $P$. uncinata, medimos: la posición del centro del tronco mayor o del centro de la mata en el caso de individuos poli- 
córmicos (coordenadas $x, y$ ), los diámetros del tronco en la base (db) y a 1,3 m sobre la base (diámetro a la altura del pecho, dbh), la altura (h), dos diámetros de la proyección vertical de la copa a lo largo de las direcciones marcadas por los ejes de la parcela (orientación aproximada N-S y W-E), altura de la rama viva más baja, número máximo de cohortes de acículas vivas (estimado mediante el examen de 4-6 ramas de la copa media de cada individuo; la longevidad de las acículas puede indicar las condiciones de crecimiento; TRANQUILLINI, 1979), y número y tipo de pies por individuo (verticalesarbóreos o arbustivos, vivos o muertos). La altura de los individuos superiores a $3 \mathrm{~m}$ fue estimada visualmente (error de medida $\pm 15 \mathrm{~cm}$; KULLMAN, 1979).

Los individuos se clasificaron según su tamaño y forma de crecimiento basándonos en una clasificación previa de BOSCH et al. (1992): dbh $>17,5 \mathrm{~cm}$, adultos (A); 7,5< dbh $<17,5 \mathrm{~cm}$, jóvenes (P, poles); dbh $<7,5 \mathrm{~cm}$ y h $>0,5 \mathrm{~m}$, vástagos ( $\mathrm{S}$, saplings); $\mathrm{h}<0,5 \mathrm{~m}$, plántulas ( $\mathrm{SE}$, seedlings); individuos muertos (D, dead trees); krummholz (K, individuos arbustivos policórmicos y de copas asimétricas); e individuos krummholz policórmicos pero con algunos pies verticales y en bandera, mostrando la mayoría de las ramas vivas orientadas en el lado opuesto al del viento más fuerte (copa asimétrica, normalmente en dirección similar a la de los krummholz) y una densa mata de pies arbustivos normamente enterrada por la nieve (KM, flagged krummholz) (SCOTT et al., 1987; GIL PELEGRÍN, 1993).

\subsection{Análisis estadísticos}

Se calculó el índice de diversidad de Shannon-Weaver $\left(\mathrm{H}\right.$, en bits $\cdot$ punto $\left.^{-1}\right)$ para las clases de individuos a lo largo del ecotono (agrupando los individuos presentes por clases cada $2 \mathrm{~m}$ a lo largo de los eje $x$ e $y$ ) (SHANNON \& WEAVER, 1949). Este índice reduce el efecto de componentes raros (MARGALEF, 1974).

Para comparar las densidades de las diferentes clases de individuos, subdividimos la parcela en una malla de 115 cuadrados de $6 \mathrm{~m} \times 6 \mathrm{~m}$ en los cuales se calculó la densidad para cada clase de individuos (número de individuos $\cdot 36 \mathrm{~m}^{-2}$ ). Esta nueva variable puede presentar autocorrelación espacial, por lo que usamos una corrección para establecer la significación exacta al relacionar densidades de distintas clases de individuos (CLIFFORD et al., 1989). Esta corrección se basa en la estimación del tamaño efectivo de muestra (p. ej. el tamaño efectivo de muestra es menor que el tamaño real en el caso de procesos con autocorrelación espacial positiva), y ha sido descrita empleando el coeficiente de correlación de Pearson $(r)$. 
La asociación entre variables estructurales se estudió usando el coeficiente no-paramétrico de correlación de Spearman $\left(r_{s}\right)$, porque la mayoría de ellas no mostraban distribuciones normales (SOKAL \& ROHLF, 1995). Para comparar medias de distintas variables estructurales (dbh, altura, etc.) entre las clases de individuos, usamos el test no-paramétrico $U$ de MannWhitney (SOKAL \& ROHLF, 1995). Para observar si existían diferencias significativas entre las distribuciones de frecuencias para variables, como el dbh y la altura, empleamos el test para dos muestras de KolmogorovSmirnov (SOKAL \& ROHLF, 1995).

\section{Resultados}

\subsection{Situación espacial en el ecotono de las clases de individuos de P. uncinata}

Los promedios de las coordenadas paralelas a la pendiente $(y)$ para las clases de individuos difirieron significativamente $(p<0,001)$, exceptuando las comparaciones con: jóvenes, vástagos, krummholz con pies verticales y muertos (Figura 6). Sin embargo, los promedios de las coordenadas perpendiculares a la pendiente $(x)$ no difirieron significativamente $(\mathrm{p}<0,001)$.

Sólo encontramos correlaciones significativas entre las coordenadas a lo largo de los ejes perpendicular $(x)$ y paralelo $(y)$ a la pendiente para los individuos jóvenes $\left(r_{s}=0,54, n=13, \mathrm{p}=0,06\right)$ y los muertos $\left(r_{s}=0,24, n=50, \mathrm{p}=\right.$ $0,1)$. Estos resultados indican cierta distribución espacial «arrinconada» para ambos tipos de individuos hacia valores altos de coordenadas $(x, y)$, o sea en la zona más occidental y próxima al bosque.

$\mathrm{Al}$, aumentar la altitud, se produce una secuencia ordenada de aparición de individuos de menor tamaño siguiendo el orden adultos $\rightarrow$ jóvenes $\rightarrow$ vástagos $\longrightarrow$ plántulas (Figura 6). Los individuos de transición tipo krummholz con pies verticales abundan entre las zonas de predominancia de individuos arbóreos (adultos, jóvenes y vástagos) y las de los arbustivos (krummholz). En las áreas más elevadas del ecotono predominan las plántulas ( $y=50$ $70 \mathrm{~m}$ ) y los krummholz $(y=30-60 \mathrm{~m})$, casi solapando sus máximos de abundancia pero siendo menos elevado el de las plántulas (Figura 6). Los individuos mayores de forma vertical y copas cónicas y simétricas (adultos y jóvenes) abundan a partir de $y=80 \mathrm{~m}$, dominado la parte inferior de la parcela, ya dentro del bosque. Los krummholz a menor elevación aparecen a $y=100-$ $105 \mathrm{~m}$ (altitud en torno a los $2095 \mathrm{~m}$ ).

Los máximos valores de diversidad corresponden al final de las formas de transición krummholz con pies verticales y el paso a la zona de dominancia 
K (404) SE (84) D (50) KM (73) S (27) P (13) A (41) Todos

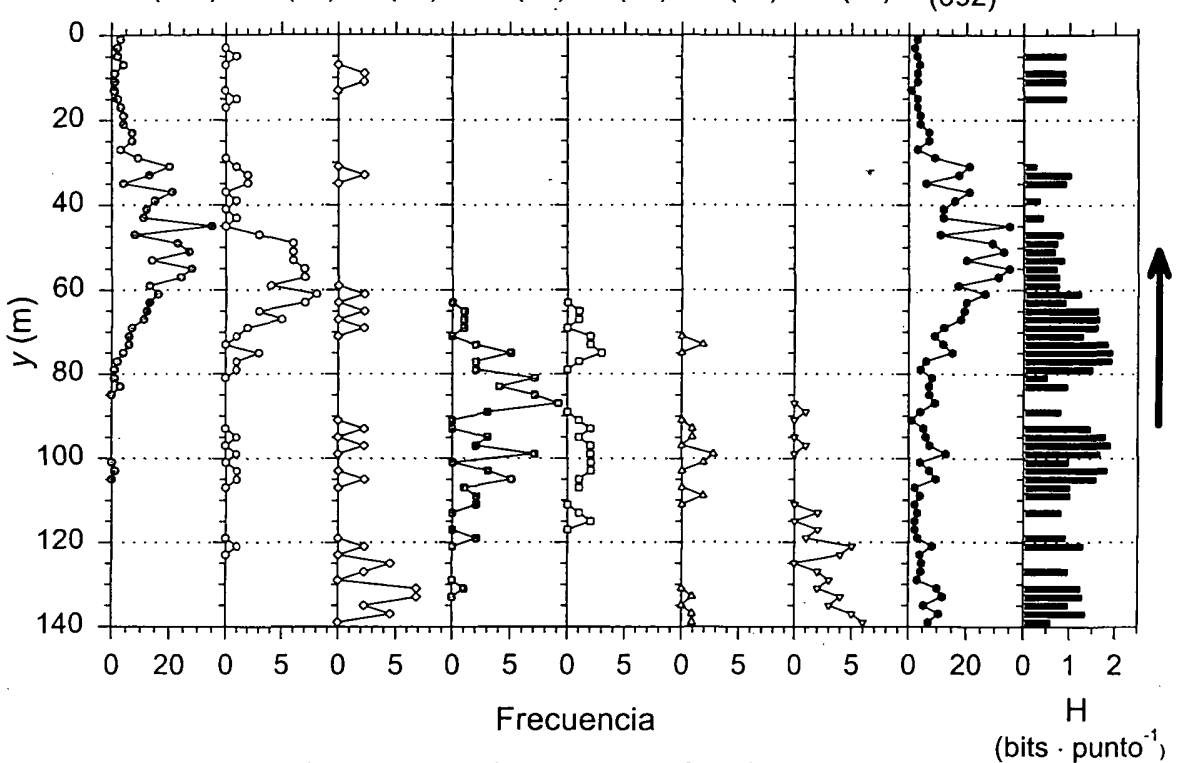

Figura 6. Frecuencia absoluta de las distintas clases de individuos de $P$. uncinata según su tamaño y forma de crecimiento y diversidad de tipos de individuos (gráfica de barras, índice $\mathrm{H}$ de Shannon-Weaver) calculados cada $2 \mathrm{~m}$ a lo largo del ecotono pastos alpinos-bosque (eje $y$ paralelo a la pendiente). Abreviaturas: A, adultos; $\mathrm{P}$, jóvenes; $\mathrm{S}$, vástagos; SE, plántulas; $\mathrm{D}$, individuos muertos; $\mathrm{K}$, krummholz; $\mathrm{KM}$, krummholz con pies verticales. La secuencia de izquierda a derecha de las clases de individuos sigue valores medios mayores de $y$, o sea menor altitud (p. ej. los individuos $\mathrm{K}$ y los $\mathrm{A}$ se sitúan, en promedio, a las altitudes más elevadas y a las menos elevadas, respectivamente, dentro del ecotono). Entre paréntesis se

indica el número de individuos de cada clase y la gráfica de símbolos negros señala la distribución de todos los individuos $(n=692)$. La flecha apunta pendiente arriba. Obsérvense las escalas distintas para los individuos krummholz y para la gráfica de todos los individuos.

Absolute frequency of the groups of P. uncinata individuals classified according to their size and growth-form and diversity of classes of individuals (bars figure, Shannon-Weaver $H$ index) calculated every $2 m$ across the subalpine forest-alpine pasture ecotone ( $y$ axis, parallel to the slope). Abbreviations: $A$, adults; $P$, poles; $S$, saplings; $S E$, seedlings; $D$, dead individuals; $K$, krummholz; KM, krummholz with vertical stems. The sequence of the classes of individuals from left to right follows greater values of $y$, i.e. lower elevation (e.g., $K$ and $A$ individuals are located, on average, at the highest and lowest elevation in the ecotone, respectively). The numbers in parenthesis are the total number of individuals for each class and the figure with black symbols shows the distribution for all the individuals $(n=692)$. The arrow points upslope. There are different scales of abundance for the figures of krummholz and all individuals.

de plántulas e individuos krummholz $(y \approx 75)$ y estos máximos son debidos al solapamiento de las tres clases antes citadas con el de aquellos individuos verticales y unicórmicos (jóvenes y vástagos), sịtuados en la mitad inferior del ecotono $(y \approx 100)$ (Figura 6). 
Los krummholz influyen decisivamente sobre la distribución de todos los individuos a lo largo de ambos ejes de la parcela $(x, y)$, ya que son la clase más abundante (constituyen el $62,93 \%$ de todos los individuos vivos y el $58,38 \%$ del total) (Figuras 6 y 7). Sólo las plántulas, los krummholz con pies verticales y, en parte, los adultos se distribuyen de modo paralelo a los krummholz a lo largo del eje perpendicular a la pendiente $(x)$, con picos de abundancia laterales y una disminución en el centro de la parcela (Figura 7). Destaca la abundancia de individuos muertos y plántulas en el lado occidental de la parcela $(x=30 \mathrm{~m})$, dirección de procedencia de los vientos dominantes. Las formas intermedias (KM), los adultos y los individuos krummholz predominan en el lado oriental $(x=0 \mathrm{~m})$. El máximo de diversidad aparece en el centro de la parcela $(x=22-24 \mathrm{~m})$, coincidiendo con abundancias elevadas de plántulas, jóvenes, muertos y krummholz con pies verticales y con valores bajos de abundancia de krummholz (Figura 7). Los máximos laterales de abundancia de todos los individuos o sólo de los krummholz en el eje menor de la parcela $(x)$ y el pico de abundancia siguiendo la pendiente $(y \approx 50 \mathrm{~m})$, reflejan lo que llamamos "cinturón de krummholz", donde se alcanzan densidades máximas dentro de la parcela (3917 árboles $\cdot$ ha $^{-1}$ para la zona $y=40-60 \mathrm{~m}$; Figura 6). Las únicas correlaciones significativas entre densidades de distintas clases de individuos de $P$. uncinata, corrigiendo la autocorrelación espacial, son aquellas entre: krummholz y plántulas $(r=0,65, \mathrm{p}<0,05)$, jóvenes y vástagos $(r=0,27, \mathrm{p}<0,05)$, y adultos y muertos $(r=0,20, \mathrm{p}<0,05)$.

Para describir la variación de altura de los individuos de $P$. uncinata a lo largo del ecotono, realizamos las figuras 8 y 9 , análogas a las presentadas por KUULUVAINEN et al. (1996). En ellas mostramos la distribución de los individuos, a lo largo de los ejes paralelo ( $y)$ y perpendicular $(x)$ a la pendiente, según su altura y teniendo en cuenta su diámetro basal, para tres límites altitudinales del bosque en los Pirineos (Las Cutas-Ordesa, Tessó del Son y Estanys de la Pera). La correlación entre la altura de los individuos vivos de $P$. uncinata y su posición a lo largo del ecotono es significativa $(\mathrm{p}<0,001)$ pero más alta en Ordesa $\left(r_{s}=0,63, n=636\right)$ que en el límite del Tessó del Son $\left(r_{s}=\right.$ $0,51, n=197)$, mientras que no lo es en los Estanys de la Pera $\left(r_{s}=-0,01, n=\right.$ $170, p=0,99$ ) (Figura 8). Se aprecian tres estructuras diferentes en cuanto a la variación de la altura de los pinos a lo largo del ecotono: (i) una muestra dos grupos claramente diferenciados situados por encima del límite del bosque (individuos bajos, altura $<0,5 \mathrm{~m}$, mayoritariamente krummholz) y dentro del bosque (individuos altos y grandes, adultos), como el caso de Ordesa; (ii) en el Tessó del Son, aparece una variación más gradual de la altura al ascender por el ecotono sin distinguirse grupos tan crontrastados aunque sí ciertas discontinuidades, y (iii) en los Estanys de la Pera, se aprecian grupos de árboles bajos y altos agregados a modo de islotes sin aparecer una clara disminución 


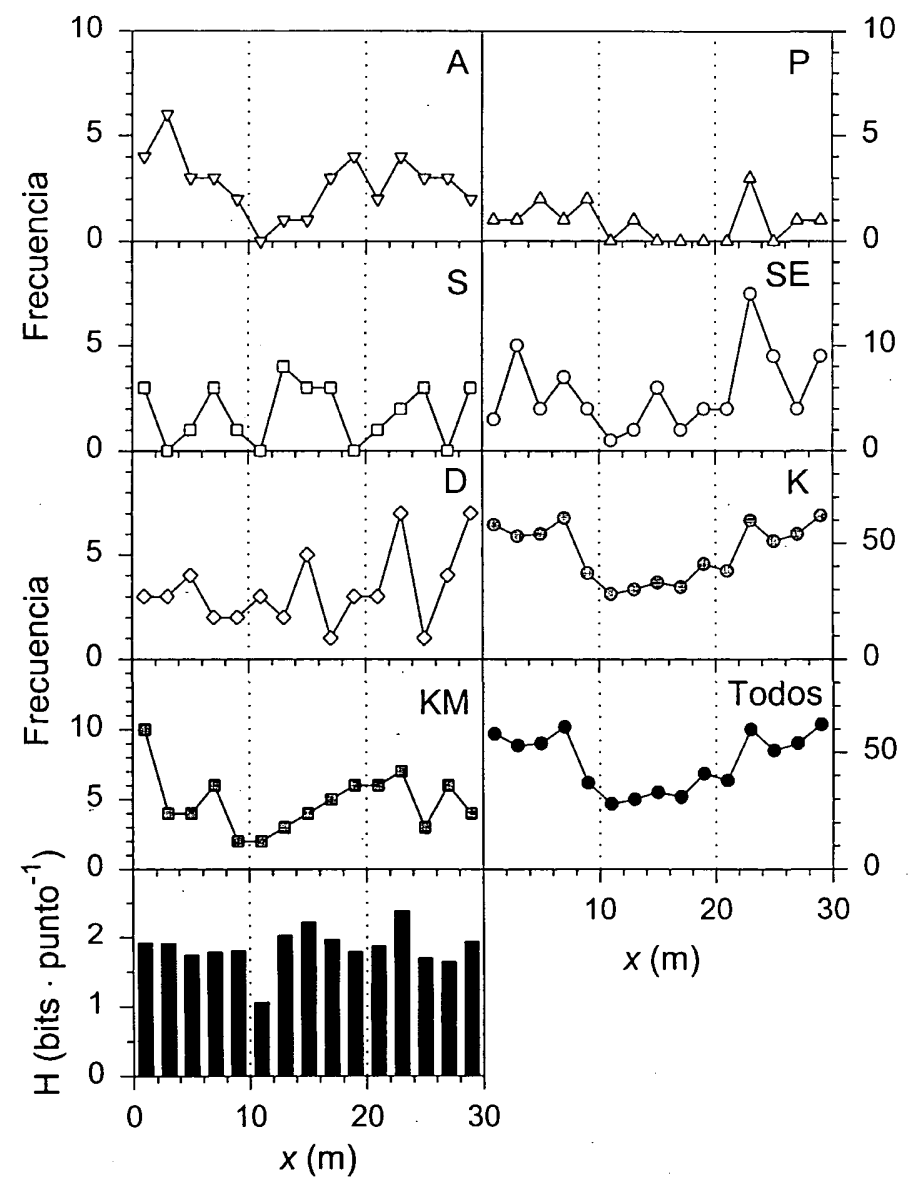

Figura 7. Frecuencia absoluta de las distintas clases de individuos de $P$. uncinata según su tamaño y forma de crecimiento y diversidad de tipos de individuos (figura de barras, índice $\mathrm{H}$ de Shannon-Weaver) calculados cada $2 \mathrm{~m}$ a lo largo del eje perpendicular a la pendiente (eje $x$ ). Abreviaturas como en la Figura 6.

Absolute frequency of the groups of P. uncinata individuals classified according to their size and growth-form and diversity of classes of individuals (bars figure, Shannon-Weaver $H$ index) calculated

every $2 m$ along the axis perpendicular to the slope ( $x$ axis). Abbreviations as in Figure 6.

de la altura a mayor elevación (Figura 8). Al observar estos mismos datos a lo largo del eje perpendicular a la pendiente $(x)$, destacan los grupos de individuos bajos (krummholz y plántulas) y altos (adultos y jóvenes) en Ordesa, la 


\section{Ordesa $\quad$ Tessó $\quad$ E. de la Pera}
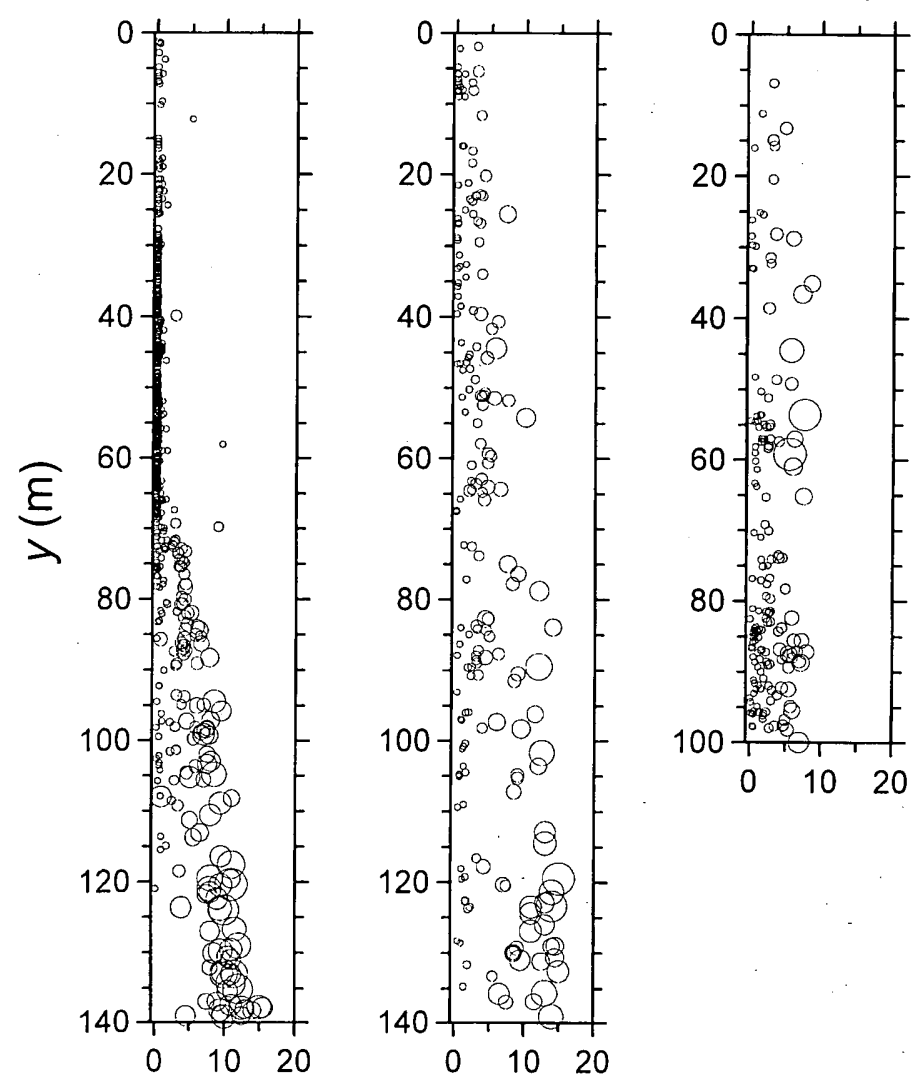

\section{Altura $(\mathrm{m})$}

Figura 8. Distribución de los individuos vivos de P. uncinata según su altura (m) a lo largo del ecotono pastos alpinos-bosque (eje de la parcela paralelo a la pendiente, $y$ ). Se comparan tres límites altitudinales del bosque en los Pirineos, dominados por P. uncinata: Las Cutas, Ordesa; Tessó, Aigüestortes i Estany de Sant Maurici y Estanys de la Pera, Lles de Cerdanya. Cada círculo muestra la altura de un individuo vivo respecto a su posición en el ecotono $(y)$ y el diámetro de los círculos es proporcional al diámetro basal del individuo.

Distribution of living P. uncinata individuals according to their height $(m)$ across the subalpine forestalpine pasture ecotone ( $y$ axis, parallel to the slope). Three altitudinal timberlines located in the Pyrenees and dominated by P. uncinata are compared: Las Cutas, Ordesa; Tessó, Aigüestortes $i$ Estany de Sant

Maurici; and Estanys de la Pera, Lles de Cerdanya. Each circle shows the height of each living individual and the position in the ecotone along the axis $y$. The circle diameter is proportional to the diameter at the base for each individual. 
aparición de individuos de distintas alturas en el Tessó del Son y una menor altura máxima para los individuos de los Estanys de la Pera en comparación con los otros dos límites forestales (Figura 9).

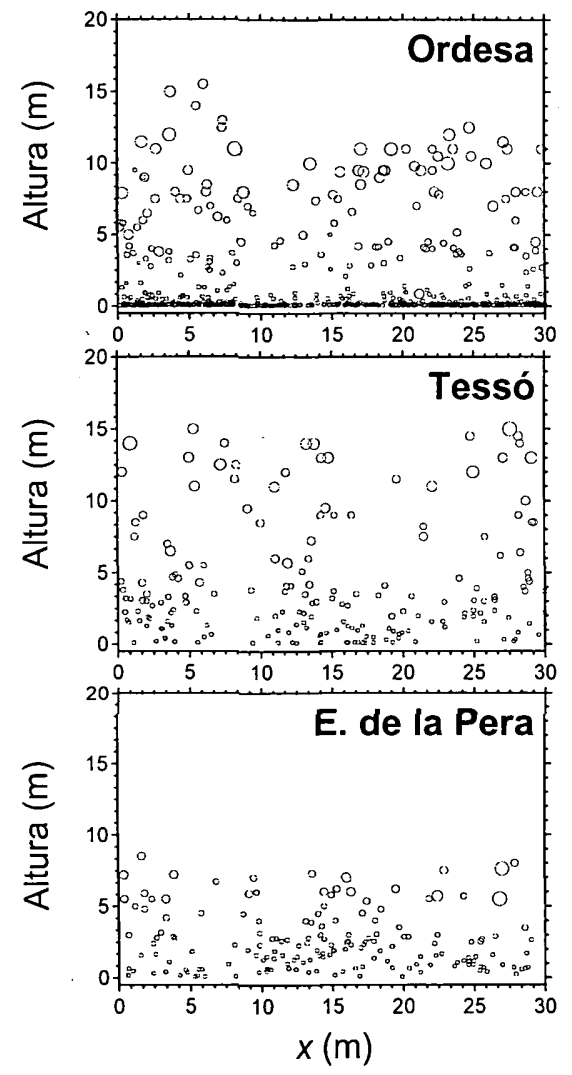

Figura 9. Distribución de los individuos vivos de $P$. uncinata según su altura $(\mathrm{m})$ a lo largo del eje de la parcela perpendicular a la pendiente $(x)$. Se comparan tres límites altitudinales del bosque en los Pirineos, dominados por P. uncinata: Las Cutas, Ordesa; Tessó, Aigüestortes i

Estany de Sant Maurici y Estanys de la Pera, Lles de Cerdanya. Cada círculo muestra la altura de un individuo vivo respecto a su posición a lo largo del eje corto de la parcela $(x)$ y el diámetro de los círculos es proporcional al diámetro basal del individuo.

Distribution of living P. uncinata individuals according to their height $(m)$ along the plot axis perpendicular to the slope $(x)$. Three altitudinal timberlines located in the Pyrenees and dominated by P. uncinata are compared: Las Cutas, Ordesa; Tessó, Aigüestortes i Estany de Sant Maurici; and Estanys de la Pera, Lles de Cerdanya. Each circle shows the height of each living individual and the position along the shortest axis of the plot $(x)$. The circle diameter is proportional to the diameter at the base for each individual. 


\section{ESTRUCTURA DE UN ECOTONO BOSQUE SUBALPINO-PASTOS ALPINOS...}

\subsection{Efectos del viento sobre las copas de los individuos de P. uncinata}

En nuestras obervaciones del límite del bosque de la Carquera, encontramos 18 individuos con un total de 96 daños en sus copas, presentando ramas muertas o erosionadas principalmente en las caras de las copas al N, NW y W (45,8\% del total de los daños, figura 10. A). Aparecen varios individuos krummholz (22\% de los individuos dañados), por encima del límite del árbol, con copas erosionadas y abundantes ramas muertas en la parte occidental de sus copas, incluso alguno forma copas en abanico con una zona cóncava al SW-W y otra convexa al NE-E, debido a la abrasión producida por los cristales de hielo arrastrados por el viento. Aparte de los individuos con daños, muchos krummholz aparecían «erosionados» en las zonas SW-W de sus copas (Figura 10. B). Otros individuos con pies verticales $(n=10)$ situados más pró-
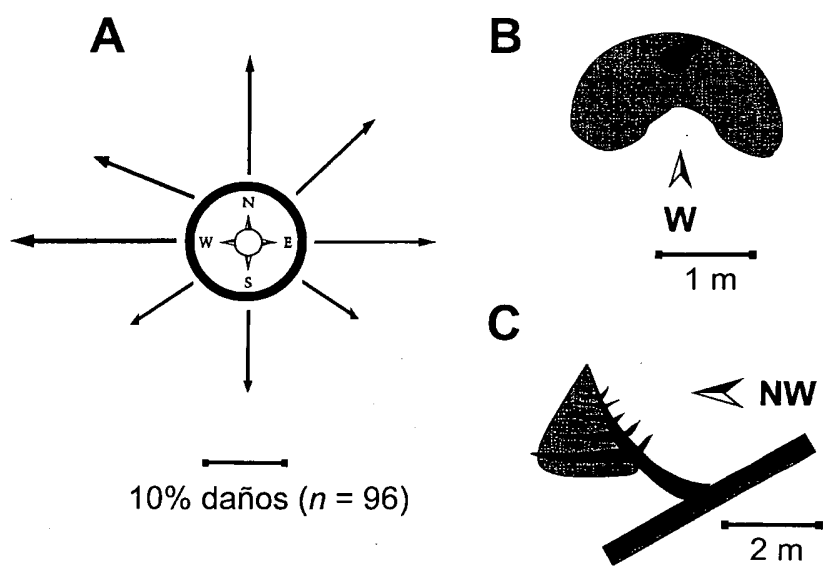

Figura 10. A. Porcentaje de daños $(n=96)$ debidos al viento, incluyendo ramas erosionadas o muertas, distribuidos en distintas orientaciones de la copa de 18 individuos de $P$. uncinata en su límite altitudinal. La barra inferior muestra la escala ( $10 \%$ de los daños). B. Proyección vertical de la copa de una forma krummholz «en cuerno o croissant» (coordenadas dentro de la parcela $(x, y)=(24,66))$. La copa está dañada por el viento en la zona occidental cóncava de la copa (el área gris es la copa y el círculo negro representa el tronco). C. Esquema de árbol bandera (coordenadas $(x, y)=(29,132)$ ) sin ramas vivas en la zona NW de la copa (la barra inferior muestra la escala y los otros símbolos son como en B).

A. Percentage of injuries $(n=96)$ due to wind effects, including abraded or dead branches, classified in the different crown directions for $18 \mathrm{P}$. uncinata individuals in the studied altitudinal timberline. The lower bar shows the scale (10\% of injuries). B. Vertical projection of the horn-shaped (or croissant-shaped) crown of a krummholz form (plot coordinates $(x, y)=(24,66)$ ). This crown is deformed (eroded) by the wind in its westerly and concave part (the gray area and the black circle represent the crown and the stem, respectively). C. Schematic representation of a flagged tree [coordinates $(x, y)=(29,132)$ ] that shows no living branch in the NW area of its crown (the lower bar shows the scale and the rest of symbols are as in $B$ ). 
ximos al límite del bosque sólo desplegaban ramas vivas hacia el E-SE (55,6\% de los individuos), típicamente en orientación $140-150^{\circ}$, o bien al SW (17\%) árboles bandera- (Figura 10. C). Las orientaciones de las copas con daños más abundantes no se corresponden con la dirección predominante del viento (Figura 4), sino con los vientos más fuertes en promedió, medidos durante todo el año (de procedencia N, W y NW; Figura 5). Sólo encontramos una correlación significativa y positiva $\left(r_{s}=0,70, n=8, \mathrm{p}=0,05\right)$ entre el número de individuos con copas dañadas por el viento clasificadas según su orientación y la velocidad media del viento medida a las $13 \mathrm{~h}$ durante todo el año. Debemos señalar que la velocidad media durante el año está significativamente correlacionada con la medida durante el invierno (abril-noviembre) a las $13 \mathrm{~h}\left(r_{s}=0,98, n=8, \mathrm{p}<0,001\right)$, como apuntaba el hecho de que las velocidades máximas registradas durante el período analizado sucedan en invierno.

\subsection{Variación de la forma de las copas de los individuos de P. uncinata}

La media ( $\pm 1 \mathrm{SD}$ ) de los diámetros de las copas de todos los individuos vivos en dirección N-S fue un poco mayor $(0,87 \pm 1,35 \mathrm{~m})$, aunque no significativamente diferente $(\mathrm{p}=0,33, n=627)$, que la medida en la dirección $\mathrm{W}$-E $(0,84 \pm 1,27$ $\mathrm{m})$. Dividiendo los individuos vivos en clases de forma y tamaño, encontramos diámetros medios significativamente $(\mathrm{p}=0,03, n=76)$ mayores en la dirección $\mathrm{W}$-E $(0,08 \pm 0,06 \mathrm{~m})$ respecto a la N-S $(0,07 \pm 0,06 \mathrm{~m})$ sólo en el caso de las plántulas. Aunque no sea significativa esta diferencia para los krummholz ( $p=0,11$, $n=401$ ), sí se mantiene la relación respecto al diámetro de las copas siendo. mayor el de la dirección W-E $(0,41 \pm 0,86 \mathrm{~m})$ respecto al N-S $(0,39 \pm 0,85 \mathrm{~m})$.

Considerando todos los individuos vivos cuyos diámetros de las copas fueron medidos $(n=580)$, la mayoría presentaron un diámetro mayor en la orientación perpendicular a la pendiente (W-E, $59,83 \%, n=347)$ frente a aquellos cuyo diámetro era mayor siguiendo la pendiente (N-S, 40,17\%, $n=$ 233). Separando los individuos por clases y formas de crecimiento y si consideramos la relación $n^{\circ}$ individuos con un diámetro de la copa mayor en dirección $\mathrm{W}$-E / n. ${ }^{\circ}$ individuos con un diámetro de la copa mayor en dirección N-S (WE / NS), este resultado se mantuvo para plántulas (WE / NS = 2,40 ), krummholz (WE / NS = 2,02) y vástagos (WE / NS = 1,36), pero no para individuos krummholz con pies verticales (WE / NS =0,59), adultos (WE / $\mathrm{NS}=0,37$ ) y jóvenes (WE / NS $=0,30$ ). Por otro lado, estas últimas tres clases de individuos son las de mayor altura media.

La relación entre la altura máxima del individuo (hmáx.) y el máximo diámetro horizontal (dmáx.) de las copas (hmáx. / dmáx.), considerando los dos diámetros medidos por individuo en direcciones W-E y N-S, es muy útil para 
describir la estructura de los individuos del ecotono del límite del bosque. Hallamos una relación hmáx / dmáx media $( \pm 1 \mathrm{SD})=1,01 \pm 0,87$. Los valores de hmáx / dmáx varían entre las distintas formas de crecimiento, siguiendo esta secuencia decreciente: jóvenes $(2,87 \pm 0,95)>$ adultos $(2,75 \pm 1,37)>$ krummholz con pies verticales $(1,83 \pm 0,82)>$ vástagos $(1,53 \pm 0,55)>$ plántulas $(0,97 \pm 0 ; 30)>$ krummholz $(0,60 \pm 0,33)$. Las comparaciones del valor medio de hmáx / dmáx entre clases o formas de crecimiento fueron significativamente $(p<0,001)$ diferentes excepto en los casos adultos-jóvenes y krummholz con pies verticales-vástagos, cuyas medias son significativamente similares $(p>0,05)$. Por lo tanto, la anterior secuencia decreciente de hmáx / dmáx podría resumirse en cuatro grupos: adultos y jóvenes (árboles verticales grandes) > krummholz con pies verticales y vástagos (formas de transición e individuos bajos) $>$ plántulas (individuos muy bajos) $>$ krummholz (formas arbustivas extendidas horizontalmente). Esta ordenación corresponde a una estructura espacial, ya que la relación entre el cociente hmáx / dmáx (variable dependiente) y la posición en el ecotono $(y)$ puede describirse bien $\left(r^{2}=0,45\right.$, $n=623, \mathrm{p}<0,001$ ) mediante una función exponencial (hmáx / dmáx $=\mathrm{a} \cdot \mathrm{e}^{\mathrm{b}}$. y), $a=0,279, b=0,017)$. En cuanto a la estructura vertical de las copas, la altura media $( \pm 1 \mathrm{SD})$ de la copa basal viva, que crece protegida bajo la nieve, de los individuos krummholz y krummholz con pies verticales era 0,74 $\pm 0,40 \mathrm{~cm}$.

\subsection{Tamaño de los individuos de P. uncinata}

Considerando la distribución de todos los individuos vivos según su diámetro a la altura del pecho (dbh), la gran abundancia de individuos krummholz y plántulas, que en su mayoría poseen $\mathrm{dbh}=0 \mathrm{~cm}$ al no alcanzar una altura de 1,3 m (Figuras 11 y 12), provoca la aparición de una distribución que puede describirse usando una función inversa (frecuencia $=\mathrm{a}+\mathrm{b} / \mathrm{dbh}, \mathrm{a}=$ $\left.15,00, \mathrm{~b}=0,05, r^{2}=0,98, n=10, \mathrm{p}<0,001\right)$. Excluyendo aquellos individuos cuyo $\mathrm{dbh}=0$, la función sencilla que mejor describe la distribución es la exponencial (frecuencia $=\mathrm{a} \cdot \mathrm{e}^{\text {(b-dbh) }}, \mathrm{a}=43,44, \mathrm{~b}=-0,07, r^{2}=0,61, n=9, \mathrm{p}<0,05$ ). A modo comparativo, la distribución de diámetros para el límite forestal del Tessó está dominada por individuos pequeños (plántulas y vástagos) pero no tanto como el caso de Ordesa (Figura 11). Así, en el Tessó la función logarítmica (frecuencia $=\mathrm{a}+\mathrm{b} \cdot \ln (\mathrm{dbh})$ ) explicó adecuadamente la distribución de diámetros para todos los individuos vivos $\left(\mathrm{a}=28,34, \mathrm{~b}=-5,56, r^{2}=0,85, n=\right.$ $15, \mathrm{p}<0,001)$ o bien sólo para aquellos cuyo dbh $>0 \mathrm{~cm}(\mathrm{a}=54,76, \mathrm{~b}=-13,19$, $\left.r^{2}=0,94, n=14, \mathrm{p}<0,001\right)$. Las distribuciones de dbh de los dos límites forestales comparados no difirieron significativamente (test de KolmogorovSmirnov, $n=10, \mathrm{p}=0,52$ ). 

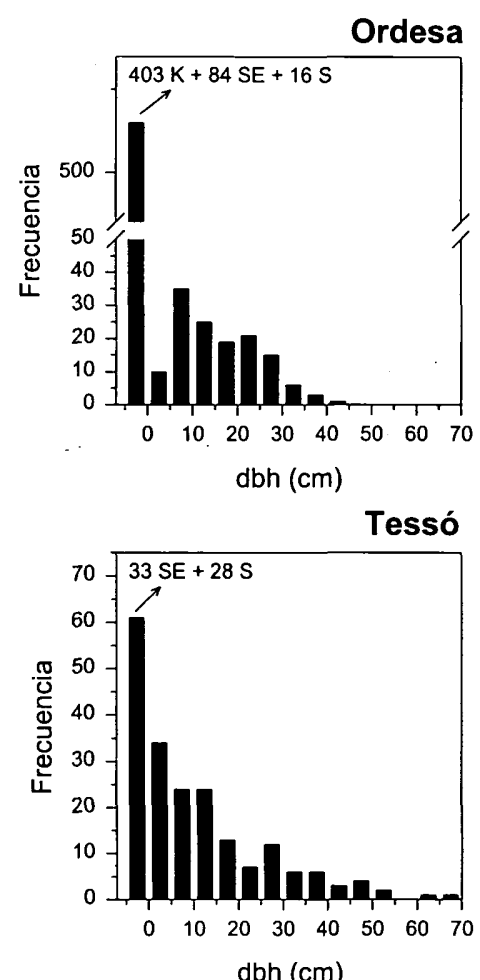

Figura 11. Distribución (frecuencia absoluta) de los individuos vivos según su diámetro a la altura del pecho (dbh, clases de $5 \mathrm{~cm}$ ) para dos límites forestales altitudinales situados en los Pirineos y dominados por $P$. uncinata: Las Cutas, Ordesa; Tessó, Aigüestortes i Estany de Sant Maurici. Se indica el número y tipo de individuos según su forma y tamaño para aquellos cuyo $\mathrm{dbh}=0 \mathrm{~cm}$ (altura $<1,3 \mathrm{~m}$ ): $\mathrm{K}$, krummholz; $\mathrm{S}$, vástagos y $\mathrm{SE}$, plántulas. Obsérvese que, en Ordesa, la mayoría de los individuos vivos cuyo $\mathrm{dbh}=0 \mathrm{~cm}$ (altura $<1,3 \mathrm{~m}$ ) son krummholz $(81,12 \%)$.

Distribution (absolute frequency). of living individuals according to their diameter at breast height (dbh, $5 \mathrm{~cm}$ classes) for two altitudinal timberlines located in the Pyrenees and dominated by P. uncinata: Las Cutas, Ordesa; and Tessó, Aigüestortes $i$ Estany de Sant Maurici. The number and type of size and growth-form class for those individuals whose $d b h=0 \mathrm{~cm}$ (height $<1.3 \mathrm{~m}$ ) is indicated: $K, \mathrm{krummholz}$; $S$, saplings; and SE, seedlings. In Ordesa, most living individuals whose $d b h=0 \mathrm{~cm}$ (height $<1.3 \mathrm{~m}$ ) were krummholz $(81.12 \%)$.

En el caso de la distribución de altura de los individuos vivos, tanto en Ordesa como en el Tessó predominaban los individuos entre 0 y 0,5 m (Figura 12). En Ordesa, otros máximoś secundarios correspondieron a los individuos de alturas comprendidas entre 3,5-4 m y 7,5-8 m. Mientras que en el Tessó aparecen frecuencias altas para los individuos de $1,5-2 \mathrm{~m}, 3-3,5 \mathrm{~m}$ y $8-9 \mathrm{~m}$. 
ESTRUCTURA DE UN ECOTONO BOSQUE SUBALPINO-PASTOS ALPINOS...
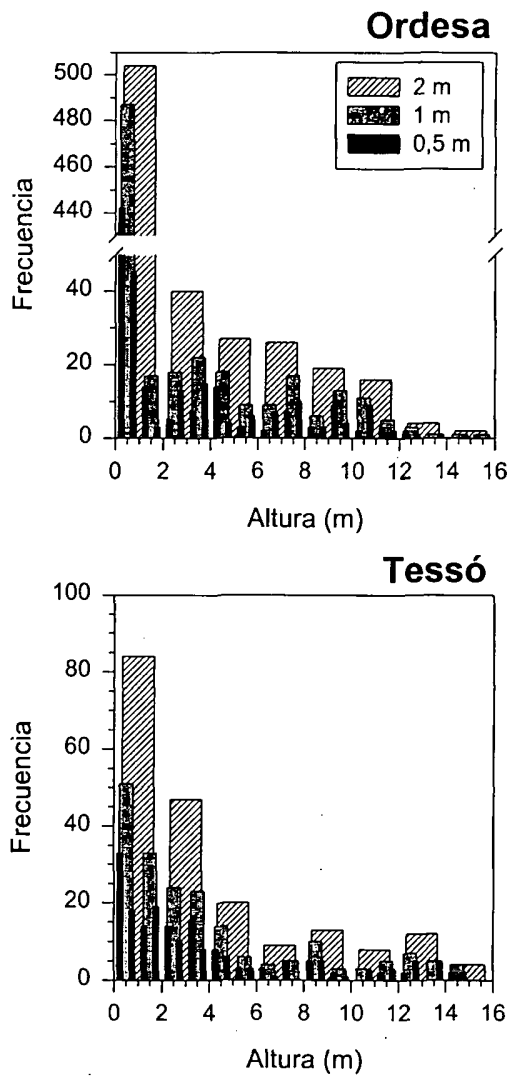

Figura 12. Distribución (frecuencia absoluta) de los individuos vivos según su altura (clases de $2 \mathrm{~m}$-barras con líneas diagonales-, $1 \mathrm{~m}$-barras grises- y 0,5 $\mathrm{m}$-barras negras-) para dos límites forestales altitudinales situados en los Pirineos y dominados por P. uncinata: Las Cutas, Ordesa; Tessó, Aigüestortes i Estany de Sant Maurici.

Distribution (absolute frequency) of living individuals according to their height $(2 \mathrm{~m}$-bars with diagonal lines-, $1 \mathrm{~m}$-gray filled bars - and $0.5 \mathrm{~m}$-black filled bars-classes) for two altitudinal timberlines located in the Pyrenees and dominated by P. uncinata: Las Cutas, Ordesa; and Tessó, Aigüestortes $i$ Estany de Sant Maurici.

En el caso de Ordesa, la función potencial describe bien la distribución de las alturas de los individuos vivos en clases de $2 \mathrm{~m}$ (frecuencia $=\mathrm{a} \cdot$ (clases-de-2m) $\left.{ }^{b}, a=466,28, b=-1,71, r^{2}=0,88, n=8, p<0,001\right)$. Sin embargo, la distribución de alturas se vuelve más compleja al subdividir las clases en rangos menores $\mathrm{y}$, aunque la función potencial es adecuada para clases de $1 \mathrm{~m}(\mathrm{a}=$ 
$110,82, \mathrm{~b}=-1,42, r=0,87, n=16, \mathrm{p}<0,001)$, la función inversa describe mejor que la potencial $\left(r^{2}=0,37\right)$ su distribución en el caso de clases de $0,5 \mathrm{~m}(\mathrm{a}=-$ $\left.15,56, \mathrm{~b}=103,79, r^{2}=0,92, n=31, \mathrm{p}<0,001\right)$. En el caso del Tessó, la función logarítmica describió el comportamiento de la distribución de alturas para clases de $2 \mathrm{~m}\left(\mathrm{a}=77,66, \mathrm{~b}=-29,21, r^{2}=0,92, n=8, \mathrm{p}<0,001\right), 1 \mathrm{~m}(\mathrm{a}=37,72$, $\left.\mathrm{b}=-14,20, r^{2}=0,92, n=15, \mathrm{p}<0,001\right)$ y $0,5 \mathrm{~m}\left(\mathrm{a}=18,67, \mathrm{~b}=-7,04, r^{2}=0,85, n\right.$ $=30, \mathrm{p}<0,001)$.

Tanto la altura media $( \pm 1 \mathrm{SD})$ de los individuos vivos de Ordesa $(1,57 \pm$ $3,02 \mathrm{~m})$ como su dbh medio $(3,44 \pm 7,92 \mathrm{~cm})$ fueron significativamente menores ( $\mathrm{p}<0,001, n=198$ y 197, respectivamente) que los valores respectivos para el ecotono del Tessó (altura media $=4,05 \pm 4,12 \mathrm{~m}$, dbh medio $=11,39 \pm 14,08$ $\mathrm{cm}$ ). Excluyendo aquellos individuos vivos cuyo $\mathrm{dbh}=0 \mathrm{~cm}$, los valores medios de dbh para Ordesa $(16,24 \pm 9,40 \mathrm{~cm})$ y Tessó $(16,46 \pm 14,25 \mathrm{~cm})$ cambian bastante y no son ahora significativamente distintos ( $\mathrm{p}=0,16, n=135)$. Los valores máximos de dbh y altura en el ecotono de Ordesa fueron $42,4 \mathrm{~cm}$ y $15,5 \mathrm{~m}$, frente a $\operatorname{los} 65,9 \mathrm{~cm}$ y $15 \mathrm{~m}$ medidos en el ecotono del Tessó. Considerando los individuos muertos, los dbh máximos medidos para éstos fueron $32 \mathrm{~cm}$ y $80 \mathrm{~cm}$ en los límites de Ordesa y el Tessó, respectivamente.

Si relacionamos la altura, el dbh y el diámetro basal (db) de los individuos vivos en ambos ecotonos (Ordesa y Tessó), los coeficientes de determinación $\left(r^{2}\right)$ más bajos siempre aparecieron al relacionar el dbh y la altura de los individuos del ecotono por lo que el db se consideró mejor predictor de la altura (Tabla 1). Todas las funciones ajustadas eran potenciales excepto las establecidas entre los diámetros medidos en la base y a la altura del pecho, que eran lineares (Figura 13). En el ecotono de Ordesa, si repetimos este análisis excluyendo los individuos krummholz, los coeficientes de determinación aumentan respecto al caso de todos los individuos vivos excepto en la regresión entre diámetros medidos en la base y a la altura del pecho. En Ordesa, en la regresión entre los diámetros medidos en la base y a la altura del pecho, el coeficiente de alometría (b) de la función potencial se aproxima a uno al excluir los krummholz, lo que indicaría una relación casi linear entre estas dos variables para los individuos no arbustivos.

En general, los coeficientes de determinación $\left(r^{2}\right)$ obtenidos para Ordesa eran siempre menores que los obtenidos en el Tessó (Tabla 1). Por ejemplo, la relación entre los diámetros medidos en la base y a la altura del pecho fue más fuerte en el Tessó que en Ordesa. El coeficiente de alometría para la regresión entre el diámetro a la altura del pecho y la altura es menor en Ordesa considerando todos los individuos (arbustivos y verticales), aumenta considerando todos los individuos en el Tessó y es aún mayor en Ordesa excluyendo los individuos con pies arbustivos (Tabla 1). 
ESTRUCTURA DE UN ECOTONO BOSQUE SUBALPINO-PASTOS ALPINOS..

\begin{tabular}{|lcccc|}
\hline Regresión $(x-y)$ & Constante $(a)$ & $\begin{array}{c}\text { Coeficiente } \\
\text { de alometria }(b)\end{array}$ & $\begin{array}{c}\text { Coeficiente } \\
\text { de determinación }\left(\mathrm{r}^{2}\right)\end{array}$ & $\begin{array}{c}\text { Número } \\
\text { de datos }(\mathrm{n})\end{array}$ \\
\hline Ordesa & & & & \\
db - altura (todos) & 0,112 & 1,141 & 0,880 & 362 \\
db-altura (sin K) & 0,169 & 1,072 & 0,916 & 182 \\
dbh-altura (todos) & 1,526 & 0,506 & 0,504 & 135 \\
dbh-altura (sin K) & 0,980 & 0,672 & 0,589 & 130 \\
db-dbh (todos) & $-2,954$ & 0,701 & 0,834 & 135 \\
db-dbh (sin K) & $-2,616$ & 0,692 & 0,827 & 130 \\
Tessó & & & & \\
db-altura & 0,302 & 0,864 & 0,931 & 194 \\
dbh-altura & 0,978 & 0,637 & 0,845 & 136 \\
db-dbh & $-3,498$ & 0,728 & 0,935 & 136 \\
\hline
\end{tabular}

Tabla 1. Parámetros y descriptivos de las regresiones ajustadas entre variables estructurales de tamaño (altura y diámetros basal $-\mathrm{db}-$ y a la altura del pecho $-\mathrm{dbh}-$ ) de los individuos de $P$. uncinata en dos ecotonos pastos alpinos-bosque: Las Cutas, Ordesa y Tessó, Aigüestortes i

Estany de Sant Maurici. En Ordesa, las regresiones se calcularon incluyendo todos los individuos vivos (todos) o excluyendo los individuos krummholz ( $\sin \mathrm{K}$ ). Todas las funciones son potenciales (p. ej. altura $=\mathrm{a} \cdot(\mathrm{dbh})^{\mathrm{b}}$ ) excepto las ajustadas entre $\mathrm{db}$ y dbh, que son lineares $(d b h=a+b \cdot d b)$. Todos los ajustes son altamente significativos $(p<0,001)$. La comparación entre los datos originales y la función ajustada para cada caso se muestra en la Figura 13, excepto para las regresiones de Ordesa realizadas excluyendo los individuos krummholz. Parameters and descriptive values of the fitted regressions among size variables (height and diameters at base - $d b$ - and breast height - dbh-) of P. uncinata individuals for two subalpine forest-alpine pasture ecotone: Las Cutas, Ordesa; and Tessó, Aigüestortes i Estany de Sant Maurici. For the Ordesa case, the regressions were calculated including all living individuals (todos) or excluding krummhoz ( $\sin K$ ). All models are power functions $\left(e . g\right.$. height $\left.=a \cdot(d b h)^{b}\right)$, excepting the linear function between $d b$ and $d b h$ $(d b h=a+b \cdot d b)$. All the fitted functions are highly signifcant $(p<0.001)$. The comparisons among raw data and the fitted function for each case are shown in the Figura 13, excepting the regressions calculated excluding krummholz individuals for the Ordesa site.

\subsection{El carácter policórmico de los individuos krummholz}

El número medio de pies por cepa o individuo fue 5. La mayoría (69\%) de los individuos eran policórmicos. El número máximo de fustes por individuo fue de 68 y se encontró en un individuo krummhoz (26 vivos y 42 muertos). El número máximo de fustes muertos por individuo fue de 42 (se trata del mismo individuo krummholz anterior). El número máximo de fustes vivos por individuo fue de 48 (46 vivos y 2 muertos); se trataba de un individuo krummholz puro. Medimos el diámetro basal de 1881 pies, de los cuales el 

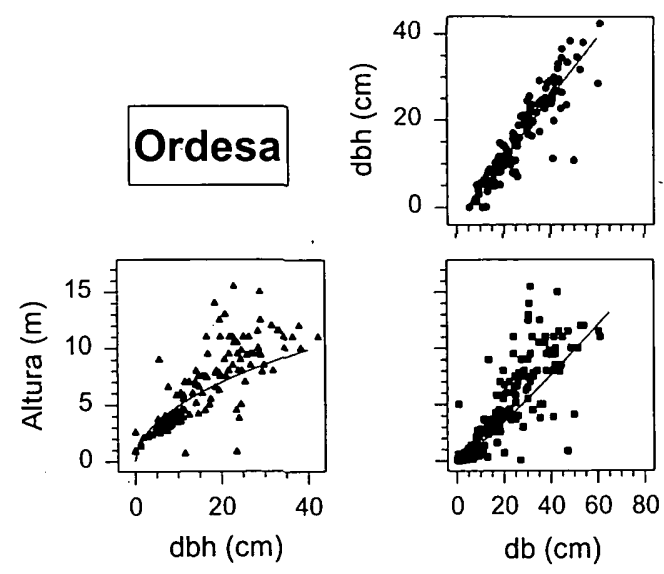

\section{Tessó}
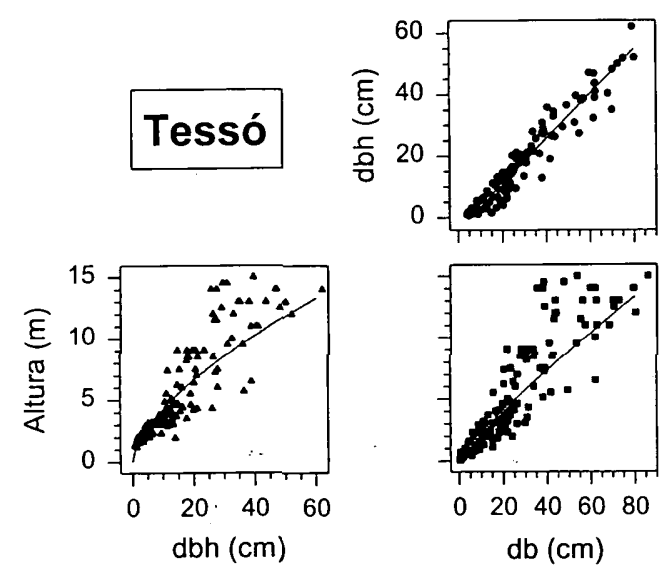

Figura 13. Regresiones entre la altura y los diámetros basal (db) y a la altura del pecho (dbh) para dos límites forestales altitudinales situados en los Pirineos y dominados por P. uncinata: Las Cutas, Ordesa; Tessó, Aigüestortes i Estany de Sant Maurici. Las líneas grises muestran la relación entre ambas variables según las funciones ajustadas (ver Tabla 1$)$. Todas las funciones son potenciales ( $p$. ej. altura $\left.=a \cdot(d b h)^{b}\right)$ excepto la ajustada entre $d b$ y $d b h$, que es linear $(d b h=a+b \cdot d b)$. Regressions among height $(m)$ and diameters at base $(d b)$ and breast height (dbh) for two altitudinal

timberlines located in the Pyrenees and dominated by P. uncinata: Las Cutas, Ordesa; and Tessó, Aigüestortes $i$ Estany de Sant Maurici. The gray lines show the relationship among variables following the functions with the best fit (see Table 1). All the models are power functions

(e. $g \cdot$ height $\left.=a \cdot(d b h)^{b}\right)$, excepting the linear function between $d b$ and $d b h(d b h=a+b \cdot d b)$.

$10,74 \%(n=202)$ eran pies muertos. La mayoría de los pies pertenecían a individuos krummholz $(71,37 \%)$ y krummholz con pies verticales $(14,51 \%)$.

El grupo más numeroso de individuos policórmicos son los individuos tipo krummholz. Considerando sólo individuos krummholz, la mayoría 
$(64,09 \%)$ de sus pies vivos mostraron diámetros basales pequeños $(<0,5 \mathrm{~cm})$ y sólo un $25,25 \%$ de sus pies muertos estaban incluidos en esta misma clase de tamaño. El número medio $( \pm 1 \mathrm{SD})$ de pies por individuo para los individuos tipo krummholz y krummholz con pies verticales fue $7 \pm 6$ y $8 \pm 9$, respectivamente. Estos resultados cambian drásticamente considerando separadamente pies vivos y muertos. Así, el número medio de pies vivos por individuo difirió significativamente entre todas las clases de individuos ( $p<0,001)$, alcanzando los valores más altos para los krummholz y los krummholz con pies verticales. No obstante, el número medio de pies muertos por individuo fue significativamente mayor $(\mathrm{p}<0,05)$ para los muertos y los individuos krummholz con pies verticales comparados con el resto de clases. En resumen, los individuos krummholz tenían muchos pies vivos por cepa pero los individuos krummholz con pies verticales y arbustivos presentaban muchos pies muertos por cepa, tantos como los individuos muertos. El resto de individuos, sobre todo los verticales y próximos al bosque (adultos, jóvenes y vástagos), tenían un número medio de pies por cepa bajo, siendo unicórmicos en su mayor parte (un $\sim 50 \%$ de las tres clases de individuos antes citadas).

Esta variación del carácter poli- / unicórmico se manifiesta a lo largo del ecotono con un incremento claro del porcentaje de pies muertos por cepa, considerando todas las clases de individuos vivos (Figura 14). Al descender por el ecotono, este cambio corresponde a un paso de la dominancia de los krummholz con muchos pies vivos por cepa a formas de transición o krummholz con pies verticales y muchos pies muertos, y, finalmente, formas unicórmicas verticales dentro del bosque (adultos y jóvenes), entre las cuales algunas presentan vestigios de krummholz como algún pie arbustivo muerto (Figs. 6 y 14). A modo de ejemplo, los dos individuos antes citados con más pies por cepa mostraron relaciones n. ${ }^{\circ}$ pies vivos / n. ${ }^{\circ}$ pies muertos iguales a $46 / 2=23$ (krummholz) y $26 / 42=0,62$ (krummholz con pies verticales).

Dicha variación del porcentaje de pies muertos o vivos por cepa respecto de la posición a lo largo del ecotono (y), se ajusta a funciones no lineares hallándose el mejor ajuste con el porcentaje de pies vivos por cepa (variable dependiente) y la posición paralela a la pendiente $(y)$. Usamos funciones cuadráticas para explicar la variación del porcentaje de pies muertos por cepa $(a=16,48, b=-0,22$, $\left.\mathrm{c}=0,004, r^{2}=0,41, n=64, \mathrm{p}<0.001\right)$ y del porcentaje de pies vivos por cepa a lo largo del ecotono $\left(\mathrm{a}=82,62, \mathrm{~b}=0,27, \mathrm{c}=-0,004, r^{2}=0,46, n=64, \mathrm{p}<0.001\right)$.

\section{Discusión}

Hemos confirmado el carácter oromediterráneo del clima y la vegetación del límite del bosque situado en Las Cutas (Ordesa). Esta característica fue ya 


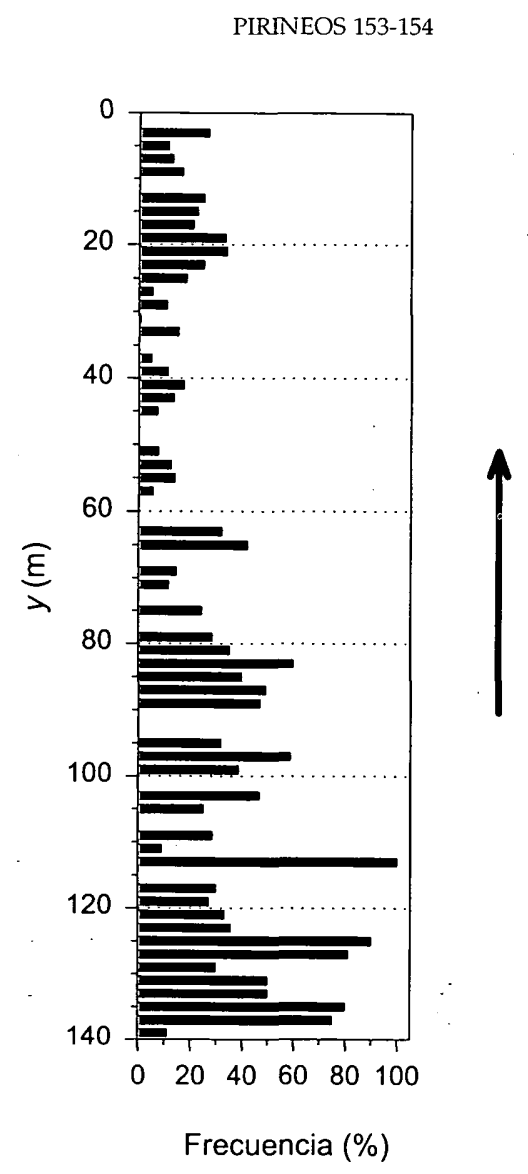

Figura 14. Aumento del porcentaje de pies muertos por cepa para todos los individuos vivos de P. uncinata cada $2 \mathrm{~m}$ al descender (valores crecientes de $y$ ) a lo largo del ecotono pastos alpinos-bosque en Ordesa. La flecha apunta pendiente arriba.

Increase of the percentage of dead stems per tree for all P. uncinata living individuals calculated every 2 $m$ descending (increasing values of $\mathrm{y}$ ) across the subalpine forest-alpine pasture ecotone in Ordesa. The arrow points upslope.

comentada por otros autores para la estación meteorológica de Góriz, situada a 2215 m s.n.m. (BALCELLS \& GIL PELEGRÍN, 1992). En este observatorio próximo, la temperatura media del mes más cálido (julio, $13^{\circ} \mathrm{C}$ ) es muy superior a los $10^{\circ} \mathrm{C}$ (Figura 1), por lo que la localización del límite del bosque estudiado (a unos $2100 \mathrm{~m}$ s.n.m.) puede explicarse mejor como un fenómeno local 
(GIL PELEGRÍN, 1993), causado en parte por la escasa innivación y permanencia de la nieve durante invierno (Figuras 2 y 3) y los fuertes vientos (Figuras 4 y 5), que basándonos en explicaciones ecofisiológicas de tipo regional como la coincidencia geográfica entre el límite del bosque y la isoterma de los $10^{\circ} \mathrm{C}$ para la temperatura media del mes más cálido (TUHKANEN, 1980; WARDLE, 1981; GRACE, 1989). Los efectos del fuerte viento son patentes en los daños de los individuos y la correlación entre la posición de los daños y la velocidad media de los vientos, que es mayor para los vientos de procedencia N, NW y W (Figs. 4, 5 y 10). El efecto del viento se manifiesta en cierta deformación de las copas a lo largo de su diámetro W-E, apareciendo acículas y ramas muertas en la dirección de procedencia del viento (NW, W), lo que genera formas «en bandera» o individuos krummholz con copas en croissant (Figura 10; GRACE, 1977).

La distribución de los individuos de $P$. uncinata a lo largo del ecotono pastos alpinos-bosque subalpino se puede considerar «ordenada» (Figura 6): Ios individuos más grandes, de copas piramidales y con pocos pies vivos por cepa aparecen dentro del bosque o cerca de su límite y, al ascender, abundan los individuos de transición (krummholz con pies verticales), las plántulas y los individuos policórmicos y arbustivos (krummholz). Esta secuencia espacial genera una zona de cambio de formas cónicas y unicórmicas (adultos, jóvenes y vástagos) a formas extendidas horizontalmente, bajas, de copas asimétricas y policórmicas (krummholz y krummholz con pies verticales), donde la diversidad de clases de individuos de P. uncinata es máxima (Figura 6). Esta estructura espacial paralela a la pendiente se hace más compleja por cambios perpendiculares a la pendiente (eje $x$ de la parcela), marcados por la abundancia de individuos krummholz que forman un «cinturón» con máximos laterales y un mínimo central (Figura 7). Se han descrito ya en los Alpes grupos densos o cinturones de krummholz (Kampfgürtel) de P. cembra, que pueden explicarse como el efecto del balance entre el crecimiento vertical durante el verano y la posterior muerte de estos nuevos pies, no del todo maduros, por desecación o daños mecánicos durante el invierno (TRANQUILLINI, 1979; WARDLE, 1981). Estos cinturones de krummholz pueden producir límites del bosque y del árbol bruscos, sin ser necesariamente de origen antrópico, caracterizados por cambios no lineares de la forma y tamaño de los individuos de P. uncinata (Figs. 8 y 14). En límites altitudinales del bosque en Chile, VEBLEN et al. (1977) describieron individuos verticales y unicórmicos de Nothofagus pumilio con restos de pies arbustivos, indicativos de su origen como formas krummholz. Nuestros resultados sugieren también la existencia de una transición morfológica y espacial, que equivale a otra temporal, desde formas arbustivas (krummholz) localizadas por encima del límite del árbol a formas arbóreas próximas al límite del bosque (adultos, jóvenes y vástagos), 
pasando por formas y posiciones intermedias (krummholz con pies verticales y pies arbustivos muertos). En otros límites forestales pirenaicos se ha mostrado ya la agregación de individuos de $P$. uncinata, algunos en forma de «cinturones» perpendiculares a la pendiente, aunque no haya sido contrastada estadísticamente (CANTEGREL, 1987).

La elevada correlación positiva entre las densidades de krummholz y plántulas debida a su proximidad espacial permite especular sobre el posible papel "protector» (nurse effect) de los krummholz sobre la regeneración de $P$. uncinata en el límite del bosque. Se ha sugerido la existencia de este efecto protector en condiciones tan limitantes como las del límite del árbol, debido al establecimiento preferencial en la periferia de individuos adultos (en nuestro caso, la regeneración preferencial se da cerca de los krummholz) y a patrones espaciales agregados o contagiosos (KULLMAN, 1983; PAYETTE \& FILION, 1985; LLOYD, 1996; CAMARERO \& GUTIÉRREZ, 1999). Esta hipótesis también se apoya en el reconocido efecto de los árboles en general y de los krummholz en particular como modificadores de su microambiente, incluido el microclima (espesor de nieve, temperatura, etc.) (HADLEY \& SMITH, 1987; HOLTMEIER \& BROLL, 1992; SCOTT et al., 1993). Una misma especie de árbol ( $P$. sylvestris) puede mostrar distintas relaciones entre clima y crecimiento según sea su forma de crecimiento (vertical o krummholz), lo que determina cómo cada forma modifica su propio microclima y sus temperaturas meristemáticas (GRACE \& NORTON, 1990).

Una característica típica de los krummholz es la compactación de sus copas que se extienden horizontalmente pero alcanzado alturas inferiores a $1 \mathrm{~m}$ (relación hmáx / dmáx baja, Figs. 8 y 9), como lo hacen plantas almohadilladas típicas de medios alpinos (WARDLE, 1968; HADLEY \& SMITH, 1986). Esta estructura permite a las formas krummholz alterar su microclima acumulando nieve en invierno y aumentando su temperatura interna durante el verano (SCOTT et al., 1993), lo que favorece la supervivencia de las acículas expuestas a la abrasión cuticular por efecto de los fuertes vientos, que arrastran cristales de hielo y nieve y posiblemente granos de arena (GRACE, 1977; HADLEY \& SMITH, 1983; 1986; 1987; TRANQUILLINI, 1979). La morfología de estos individuos arbustivos está muy relacionada con la variación de las condiciones locales limitantes (NORTON \& SCHÖNENBERGER, 1984; SCOTT et al., 1987), lo cual ha servido para su uso paleoecológico, p. ej. como reconstructores de cambios en el espesor de nieve (LAVOIE \& PAYETTE, 1992).

Las distribuciones de diámetro a la altura del pecho $(\mathrm{dbh})$ para los individuos vivos sugieren una mortalidad constante en los dos ecotonos comparados (Figura 11), aunque un reclutamiento más continuo o una distribución más equitativa de las clases de tamaño en el Tessó que en Ordesa (HETT \& LOUCKS, 1976). Ambas poblaciones serían demográficamente 
inestables, como lo muestra la dificultad de ajustar funciones potenciales tanto a las distribuciones de diámetro a la altura del pecho como a las de alturas debido a la predominancia de individuos pequeños aunque no necesariamente jóvenes (p. ej. los individuos krummholz en Ordesa, figs. 11,12 y 13). Sólo las frecuencias elevadas de $\mathrm{dbh}=20-30 \mathrm{~cm}$ y $45-50 \mathrm{~cm}$ (sólo en el Tessó) muestran valores similares a los de otros estudios de límites forestales de los Pirineos franceses (CANTEGREL, 1987). No obstante, todas estas afirmaciones deben ser confirmadas por distribuciones de edades y por estudios dendroecológicos que describan el componente temporal. Las densidades estimadas de individuos pequeños $(\mathrm{dbh}=0 \mathrm{~cm})$ son muy elevadas en Ordesa (1198 individuos $\cdot \mathrm{ha}^{-1}$ ) en comparación con otros ecotonos bosque subalpino-pastos alpinos de los Pirineos franceses (Anie, 180 individuos $\cdot$ ha $^{-1}$; Néouvielle-Estibère, 203 individuos $\cdot$ ha $^{-1}$; CANTEGREL, 1987) debido a la abundancia de individuos krummholz, aunque los valores del Tessó son similares o incluso inferiores a estos datos (145 individuos $\left.\cdot \mathrm{ha}^{-1}\right)$.

Los coeficientes de determinación al comparar los diámetros medidos en la base y a la altura del pecho entre sí y con la altura de los árboles fueron menores para Ordesa que para el Tessó debido a la abundancia de individuos tipo krummholz en el primer ecotono. El crecimiento en altura, para un mismo incremento del diámetro a la altura del pecho, es mayor para los individuos no arbustivos de Ordesa y del Tessó, pero es menor para todos los individuos de Ordesa, que incluyen los arbustivos. La abundancia de individuos arbustivos y el efecto del viento en el ecotono de Ordesa cambian las relaciones alométricas entre diámetros y altura, provocando menores crecimientos en altura para un mismo incremento de diámetro a la altura del pecho. En el ecotono de Ordesa y al relacionar diámetro basal y altura, el coeficiente de alometría (Tabla 1, b), próximo a uno excluyendo los krummholz -isometría - o superior a ùno incluyendo todos los individuos, indica mayores crecimientos en altura para un mismo aumento de diámetro basal que en el Tessó (Tabla 1). Dicho de otro modo, para alturas inferiores o iguales a $\sim 6,5 \mathrm{~m}$, los individuos de Ordesa muestran diámetros basales mayores que los individuos del Tessó de igual altura. La relación entre diámetro basal y altura se invierte para valores de altura mayores de $6,5 \mathrm{~m}$, valor en el cual se cruzarían las curvas de las funciones alométricas negativa (Tessó, $b<1$ ) y positiva (Ordesa, $b>1$ ). El engrosamiento basal de los individuos de Ordesa puede explicarse por su origen o condición arbustiva y policórmica. Sus múltiples pies basales pueden deberse a la abrasión del viento y los cristales de nieve y el daño continuo que producen sobre los tejidos meristemáticos, lo que originaría callos cuyos tejidos pueden diferenciarse produciendo nuevos pies (TRANQUILLINI, 1979). 
La variación a lo largo del ecotono de varias características estructurales no fue linear (p. ej. el número de pies muertos por cepa de P. uncinata, figura 14). Esto refuerza la hipótesis sobre el carácter local del ecotono estudiado (GIL PELEGRÍN, 1993), que podría explicarse por las estresantes condiciones locales ambientales (acentuados contrastes térmicos, fuertes vientos, escasa protección por la nieve, etc.). El carácter local del ecotono estudiado debe considerarse en el contexto histórico de la zona, con una larga tradición de uso de los pastos alpinos (ALDEZÁBAL et al., 1992). La importancia de las condiciones climáticas locales y la transición espacial de las distintas formas de crecimiento a lo largo del ecotono sugieren que los cambios climáticos, presentes o pasados, son la causa principal que explica los procesos de crecimiento y regeneración y el patrón estructural descrito en el límite del bosque estudiado. La gestión de los límites del bosque pirenaicos debe considerar los cambios climáticos actuales como agente causal determinante de la estructura del límite del bosque. Para ello es preciso conocer la ecología y reconstruir la paleoecología de estos ecotonos a través de técnicas como la dendroecología.

La existencia de individuos verticales unicórmicos con rasgos relictuales de individuo arbustivo policórmico (p. ej., restos de pies arbustivos) en el ecotono estudiado demuestra que la transición forma arbórea $\leftrightarrow$ forma arbustiva es posible, como muestran las formas intermedias con pies verticales por encima de la mata arbustiva. Estos cambios morfológicos y el posible efecto protector de individuos arbustivos (krummholz) sobre la regeneración pueden ser factores básicos que produzcan cierta inercia frente a cambios climáticos adversos. Esta inercia implicaría que un cambio climático favorable puede producir ascensiones relativamente rápidas del límite del árbol mientras que un cambio adverso puede no generar retrocesos altitudinales (KULLMAN, 1990; LLOYD, 1996), debido a la versatilidad fenotípica de los árboles y a interacciones intraespecíficas tipo facilitación que han sido descritas en otros medios (p. ej. pastos alpinos, tundras y zonas áridas), igualmente bajo condiciones ambientales muy adversas (BERTNESS \& CALLAWAY, 1994; KIKVIDZE \& NAKHUTSRISHVILI, 1998).

Agradecimientos. Este trabajo forma parte de un informe para el Parque Nacional de Ordesa y Monte Perdido, cuyo personal y dirección nos prestaron el apoyo y permisos necesarios. Gracias por su colaboración en el muestreo a: J. L. Benito Alonso, A. Bernat, E. Muntán, M. Ribas y R. Romano. Gracias por la ayuda prestada a: Jesús (el guarda forestal de Alagón), J: Bas, M. Etxebarría y E. Gil Pelegrín. Este trabajo ha sido financiado por el Ministerio de Educación y Ciencia a través de un proyecto CICyT (AMB950160) y una beca F. P. I. al primer autor (AP93 72784356). Los datos climáticos fueron facilitados por el Centro Territorial de Aragón, La Rioja y Navarra del Instituto Nacional de Meteorología. 
ESTRUCTURA DE UN ECOTONO BOSQUE SUBALPINO-PASTOS ALPINOS...

\section{Referencias}

ALDEZÁBAL, A.; BAS, J.; FILLAT, F.; GARCÍA GONZÁLEZ, R.; GARIN, I.; GÓMEZ, D. \& SANZ, J. L. (1992). Utilización ganadera de los pastos supraforestales en el Parque Nacional de Ordesa y Monte Perdido. Informe Consejo Superior̀ de Investigaciones Científicas - Instituto Nacional para la Conservación de la Naturaleza, 149 pp., Jaca.

ALLUÉ ANDRADE, J. L. (1990). Atlas Fitoclimático de España. Taxonomías. Colección Monografías I.N.I.A., 69. Ministerio de Agricultura, Pesca y Alimentación, 221 pp., Madrid.

BALCELLS, E. \& GIL PELEGRÍN, E. (1992). Consideraciones fenológicas de las biocenosis de altitud en el Parque Nacional de Ordesa y Monte Perdido, acompañadas y apoyadas mediante estudio preliminar de los datos meteorológicos obtenidos desde 1981 a 1989 en el observatorio de Góriz. Lucas Mallada, 4: 71-162.

BARRIO, G. del; CREUS, J. \& PUIGDEFÁBREGAS, J. (1990). Thermal seasonality of high mountain belts of the Pyrenees. Mount. Res. Dev., 10 (3): 227233.

BAS i CASAS, J. (1993). Les pastures supraforestals a la Vall Ferrera i la Vall de Cardós (Pallars Sobirà). Valoració de la capacitat ramadera de les pastures de Lladorre. Projecte Final de Carrera. Escola Tècnica Superior d'Enginyeria Agrària de Lleida, Universitat de Lleida.

BERTNESS, M. D. \& CALLAWAY, R. (1994). Positive interactions in communities. TREE, 9: 191-193.

BOLÒS, O. de; VIGO, J.; MASALLES, R. M. \& NINOT, J. M. (1993). Flora manual dels Països Catalans. Ed. Pòrtic, 1247 pp., Barcelona.

BOSCH, O.; GINÉ, L.; RAMADORI, E. D.; BERNAT, A. \& GUTIÉRREZ E. (1992). Disturbance, age and size structure in stands of Pinus uncinata Ram. Pirineos, 140: 5-14.

BRINK, V. C. (1959). A directional change in the subalpine forest-heath ecotone in Garibaldi Park, British Columbia. Ecology, 40: 10-16.

BRUBAKER, L. B. (1986). Responses of tree populations to climatic change. Vegetatio, 67: 119-130.

BÜCHER, A. \& DESSENS, J. (1991). Secular trend of surface temperature at an elevated observatory in the Pyrenees. J. Climatology, 4: 859-868.

CAMARERO, J. J. \& GUTIÉRREZ, E. (1999). Patrón espacial de un ecotono bosque subalpino-pastos alpinos (Las Cutas, Ordesa, Pirineos Centrales). INIA - Sist. Recur. For., 8 (1): 171-205.

CANTEGREL, R. (1983). Le Pin à crochets pyrénéen: biologie, biochimie, sylviculture. Acta Biologica Montana, 2-3: 87-330. 
CANTEGREL, R. (1987). Productivité ligneuse et organisation des marges forestières à Pinus uncinata Ram. en Pyrénées Occidentales. Pirineos, 130: 3-27.

CARRERAS, J.; CARRILLO, E.; MASALLES, R. M.; NINOT, J. M.; SORIANO, I. \& VIGO, J. (1996). Delimitation of the supra-forest zone in the Catalan Pyrenees. Bull. Soc. Linn. Provence, 47: 27-36.

CEBALLOS y FERNÁNDEZ DE CÓRDOBA, L. \& RUIZ de la TORRE, J. (1979). Árboles y Arbustos de la España Peninsular. Ed. Escuela Técnica Superior de Ingenieros de Montes, 512 pp., Madrid.

CLIFFORD, P.; RICHARDSON, S. \& HÉMON, D. (1989). Assesing the significance of the correlation between two spatial processes. Biometrics, 45: 123-134.

CREUS, J. (1978): Influencias del relieve en la distribución de las precipitaciones máximas. Cuadernos de Investigación, 4: 11-23.

CHOCARRO, C.; FANLO, R.; FILLAT, F. \& MARIN, P. (1990). Historical evolution of natural resource use in the Central Pyrenees of Spain. Mount. Res. Dev., 10 (3): 257-265.

DESSENS, J. \& BÜCHER, A. (1997). A critical examination of the precipitation records at the Pic du Midi observatory, Pyrenees, France. Clim. Change, 36: 345-353.

DUNWIDDIE, P. W. (1977). Recent tree invasion of subalpine meadows in the Wind River Mountains, Wyoming. Arct. Alp. Res., 9: 393-399.

FRANKLIN, J. F.; MOIR, W. H.; DOUGLAS, G. W. \& WIBERG, C. (1971): Invasion of subalpine meadows by trees in the Cascade Range, Washington and Oregon. Arct. Alp. Res., 3: 215-224.

FREY, W. (1983). The influence of snow on growth and survival of planted trees. Arct. Alp. Res., 15: 241-251.

GARCÍA RUIZ, J. M. \& LASANTA MARTÍNEZ, T. (1990). Land-use changes in the Spanish Pyrenees. Mount. Res. Dev., 10 (3): 267-279.

GIL PELEGRÍN, E. (1993). Estudios ecofisiológicos sobre Pinus uncinata Mill. en el límite superior de, la especie. Tesis Doctoral, Fac. Ciencias, Depto. de Ecología, Universidad Autónoma de Madrid, 231 pp., Madrid.

GIL PELEGRÍN, E. \& VILLAR, L. (1988). Structure of mountain pine (Pinus uncinata) populations at its upper limit in Central Pyrenees. Pirineos, 131: 25-42.

GRACE, J. (1977). Plant Response to Wind. Academic Press, 204 pp., London.

GRACE, J. (1989). Tree lines. Phil. Trans. R. Soc. London S. B, 324: 233-245.

GRACE, J. \& NORTON, D. A. (1990). Climate and growth of Pinus sylvestris at its upper altitudinal limit in Scotland: evidence from tree growth-rings. J. Ecol., 78: 601-610. 
HADLEY, J. L. \& SMITH, W. K. (1983). Influence of wind exposure on needle dessication and mortality for timberline conifers in Wyoming, USA. Arct. Alp. Res., 15: 127-135.

HADLEY, J. L. \& SMITH, W. K. (1986). Wind effects on needles of timberline conifers: seasonal influence on mortality. Ecology, 67: 12-19.

HADLEY, J. L. \& SMITH, W. K. (1987). Influence of krummholz mat microclimate on needle physiology and survival. Oecologia, 73: 82-90.

HANSEN, A. \& di CASTRI, F. (eds.). (1992). Landscape boundaries: consequences for biotic diversity and ecological flows. Springer-Verlag, New York.

HETT, J. M. \& LOUCKS, O. L. (1976). Age structure models of balsam fir and eastern hemlock. J. Ecol., 64: 1029-1044.

HOLTMEIER, F.-K. \& BROLL, G. (1992). The influence of tree islands and microtopography on pedoecological conditions in the forest-alpine tundra ecotone on Niwot Ridge, Colorado Front Range, U. S. A. Arct. Alp. Res., 24: 216-228.

IVES, J. D. \& HANSEN-BRISTOW, K. J. (1983). Stability and instability of natural and modified upper timberline landscapes in the Colorado Rocky Mountains, USA. Mount. Res. Dev., 3: 149-155.

JAKUBOS, B. \& ROMME, W. H. (1993). Invasion of subalpine meadows by Lodgepole pine in Yellowstone National Park, Wyoming, U.S.A. Arct. Alp. Res., 25: 382-390.

KEARNEY, M. S. (1982). Recent seedling establishment at timberline in Jasper National Park, Alberta. Can. J. Bot., 60: 2283-2287.

KIKVIDZE, Z. \& NAKHUTSRISHVILI, G. (1998). Facilitation in subnival vegetation patches. J. Veg. Sci., 9: 261-264.

KULLMAN, L. (1979). Change and stability in the altitude of the birch treelimit in the southern Swedish Scandes 1915-1975. Acta Phytogeographica Suecica, 65: 1-121.

KULLMAN, L. (1983). Short-term population trends of isolated tree-limit stands of Pinus sylvestris L. in central Sweden. Arct. Alp. Res., 15: 369-382.

KULLMAN, L. (1990). Dynamics of altitudinal tree-limits in Sweden: a review. Norsk Geografisk Tidsskrift, 44: 103-116.

KULLMAN, L. (1991). Pattern and process of present tree-limits in the Tärna region, southern Swedish Lapland. Fennia, 169 (1): 25-38.

KUULUVAINEN, T.; PENTTINEN, A.; LEINONEN, K. \& NYGREN, M. (1996). Statistical opportunities for comparing stand structural heterogeneity in managed and primeval forests: an example from boreal spruce forest in Southern Finland. Silva Fennica, 30: 315-328.

LAVOIE, C. \& PAYETTE, S. (1992). Black spruce growth forms as a record of a changing winter environment at tree line, Québec, Canada. Arct. Alp. Res., 24: 40-49. 
LLOYD, A. H. (1996). Patterns and processes of treeline response to late Holocene climate change in the Sierra Nevada, California. PhD Dissertation, Dept. of Ecology and Evolutionary Biology, The University of Arizona, 184 pp., Tucson, Arizona, U.S.A.

MARGALEF, R. (1974). Ecología. Ed. Omega, 951 pp., Barcelona.

MIROV, N. T. (1967). The genus Pinus. The Ronald Press Co., 602 pp., New York.

MONTERO DE BURGOS, J. L. \& GONZÁLEZ REBOLLAR, J. L. (1974). Diagramas bioclimáticos. Instituto Nacional para la Conservación de la Naturaleza, 379 pp., Madrid.

MONTSERRAT, J. M. (1992). Evolución glaciar y postglaciar del clima y la vegetación en la vertiente Sur del Pirineo: estudio palinológico. Ed. Consejo Superior de Investigaciones Científicas (C. S. I. C.), Monografías del Instituto Pirenaico de Ecología, n 6., 152 pp., Zaragoza.

NORTON, D. A. \& SCHÖNENBERGER, W. (1984). The growth forms and ecology of Nothofagus solandri at the alpine timberline, Craigieburn Range, New Zealand. Arct. Alp. Res, 16: 361-370.

OKSANEN, L.; MOEN, J. \& HELLE, T. (1995). Timberline patterns in northernmost Fennoscandia. Acta Botanica Fennica, 153: 93-105.

PAYETTE, S. \& FILION, L. (1985). White spruce expansion at the tree line and recent climatic change. Can. J. For. Res., 15: 241-251.

PAYETTE, S. \& LAVOIE, C. (1994). The arctic tree line as a record of past and recent climatic changes. Env. Rev., 2: 78-90.

RÍOS, L. M.; LANAJA, J. M. \& FRUTOS, E. (1982). Mapa geológico de España. E. 1: 50.000. Hoja 178 (Broto). Ed. Instituto Geológico y Minero de España, 60 pp., Madrid.

SCOTT, P. A.; BENTLEY, C. V.; FAYLE, D. C. F. \& HANSELL, R. I. C. (1987). Crown forms and shoot elongation of white spruce at the treeline, Churchill, Manitoba, Canada. Arct. Alp. Res., 19: 175-186.

SCOTT, P. A.; HANSELL, R. I. C. \& ERICKSON, W. R. (1993). Influences of wind and snow on Northern tree-line environments at Churchill, Manitoba, Canada. Arctic, 46: 316-323.

SHANNON, C. E. \& WEAVER, W. (1949). The mathematical theory of communication. The University of Illinois Press, 125 pp., Urbana, USA.

SOKAL, R. R. \& ROHLF, F. J. (1995). Biometry: the principles and practice of statistics in biological research. W. H. Freeman \& Co., 887 pp., New York.

SOUTADÉ, G.; BAUDIÈRE, A. \& BÉCAT, J. (eds.) (1982). La limite supérieure de la forêt et sa valeur de seuil. Ed. Terra Nostra, 174 pp.., Prada de ConflentPerpignan.

TRANQUILLINI, W. (1979). Physiological ecology of the alpine timberline. Springer-Verlag, 137 pp., Berlin. 
ESTRUCTURA DE UN ECOTONO BÓSQUE SUBALPINO-PASTOS ALPINOS...

TUHKANEN, S. (1980). Climatic parameters and indices in plant geography. Acta Phytogeographica Suecica, 67: 1-105.

VALE ,T. R. (1977). Forest changes in the Warner Mountains, California. Ann. Ass. Am. Geogr., 67: 28-45.

VALE, T. R. (1987). Vegetation change and park purposes in the high elevations of Yosemite National Park, California. Ann. Ass. Am. Geogr., 77: 1-18.

VEBLEN, T. T.; ASHTON, D. H.; SCHLEGEL, F. M. \& VEBLEN, A. T. (1977). Plant succession in a timberline depressed by vulcanism in south-central Chile. J. Biogeogr., 4: 275-294.

VIGO i BONADA, J. (1976). L'alta muntanya catalana. Flora $i$ vegetació. Ed. Montblanc-Martin, 421 pp., Barcelona.

VILLAR, L. \& BENITO ALONSO, J. L. (1994). Esquema de la vegetación del Parque Nacional de Ordesa y Monte Perdido, más su zona periférica. Lucas Mallada, 6: 235-273.

VILLAR, L. \& BENITO ALONSO, J. L. (1996). Riqueza de la vegetación del Parque Nacional de Ordesa y Monte Perdido en relación con los hábitats de interés europeo. Tomo Extraordinario, XII Bienal, 125 Aniversario de la Real Sociedad Española de Historia Natural, pp. 459-464.

VILLAR, L. \& MONTSERRAT RECODER, P. (1990). Guía de la excursión Jaca-Ordesa (5 de julio de 1989). In VILLAR, L. (ed.). Botánica PirenaicoCantábrica, Instituto de Estudios Altoaragoneses - Instituto Pirenaico de Ecología (C.S.I.C.): 707-729. Huesca y Jaca.

VITOUSEK, P. M. (1994). Beyond global warming: ecology and global change. Ecology, 75: 1861-1876.

WARDLE, P. (1968). Engelmann spruce (Picea engelmannii Engel.) at its upper limits on the Front Range, Colorado. Ecology, 49: 483-495.

WARDLE, P. (1981). Is the alpine timberline set by physiological tolerance, reproductive capacity, or biological interactions? Proc. Ecol. Soc. Aust. 11: 53-66. 